A

West of Wrangell
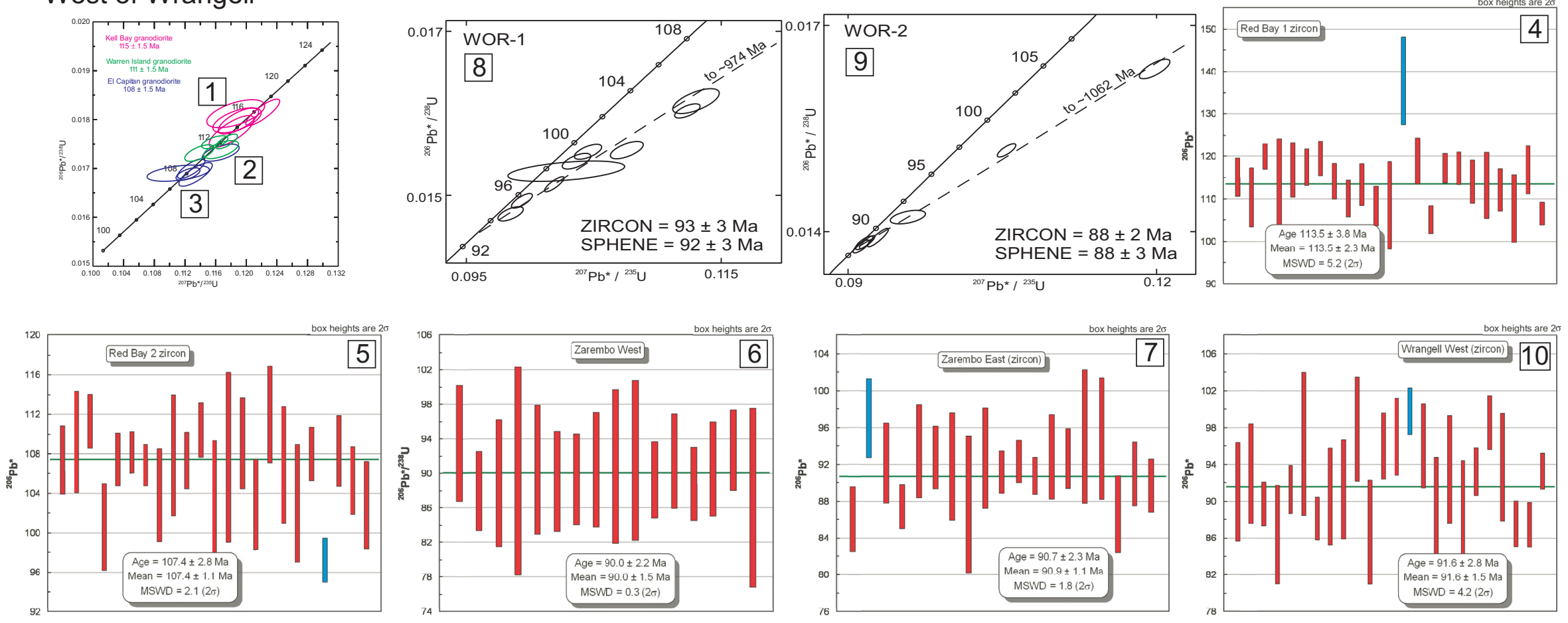

\title{
East of Wrangell
}
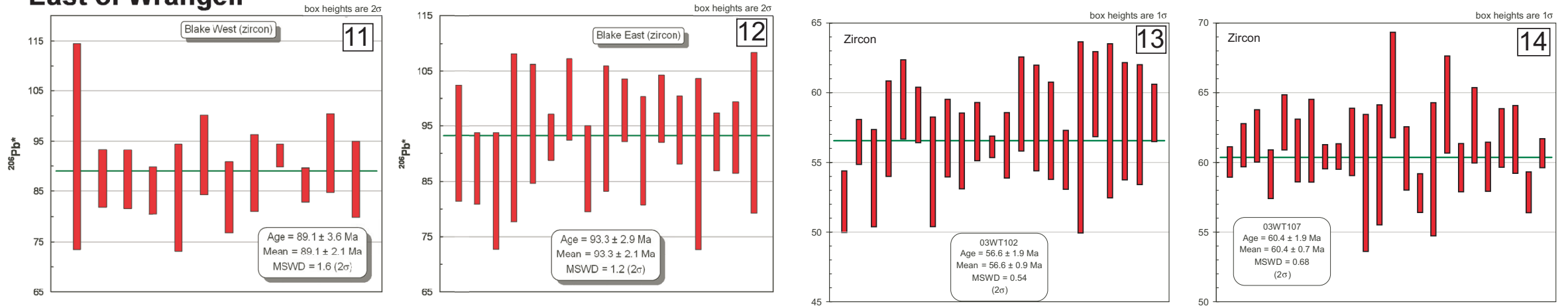

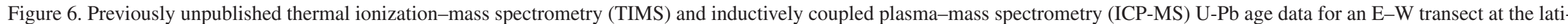

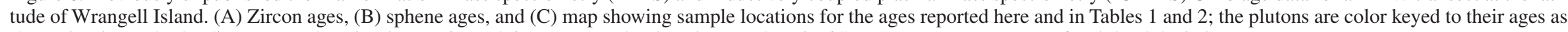
shown in Figure 5. The diagram numbers in Figures 6A and 6B correspond to location numbers in 6C. MSWD-mean square of weighted deviations. 
B

\section{West of Wrangell}

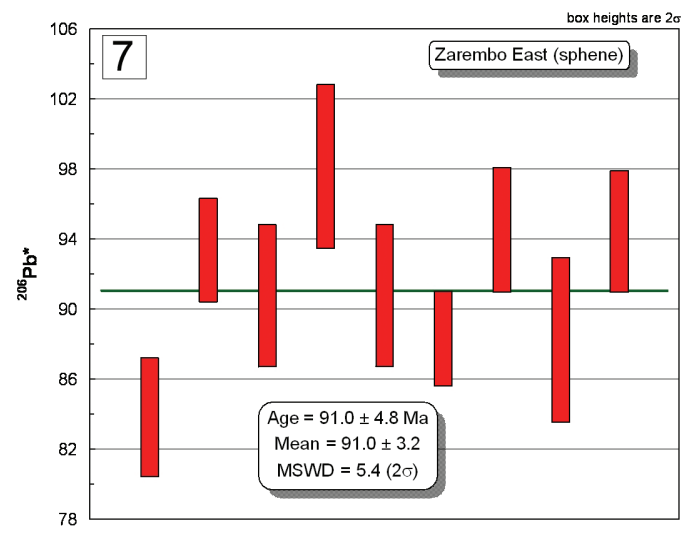

\section{East of Wrangell}
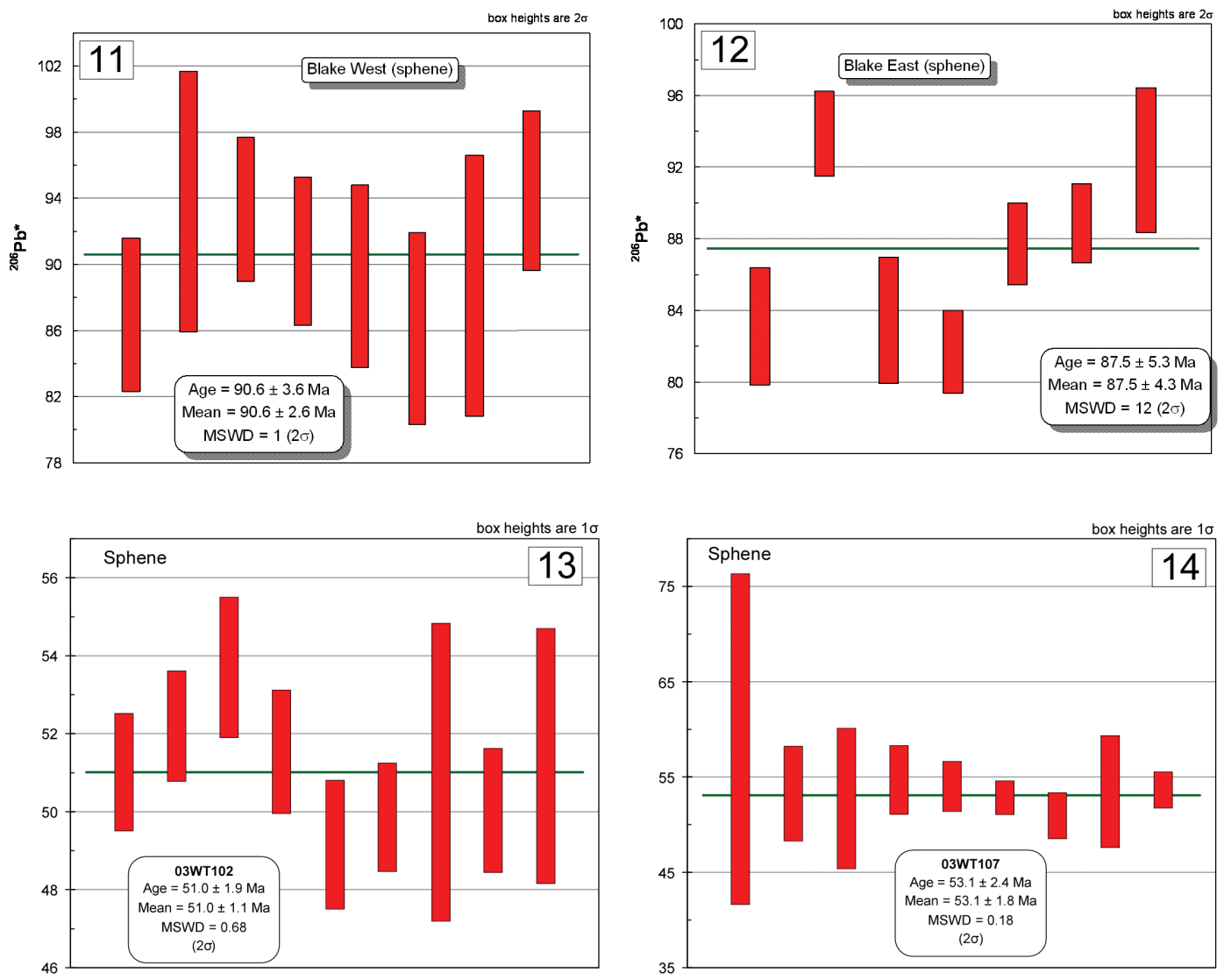

Figure 6. (Continued.) 
C $135^{\circ} \mathrm{W}$

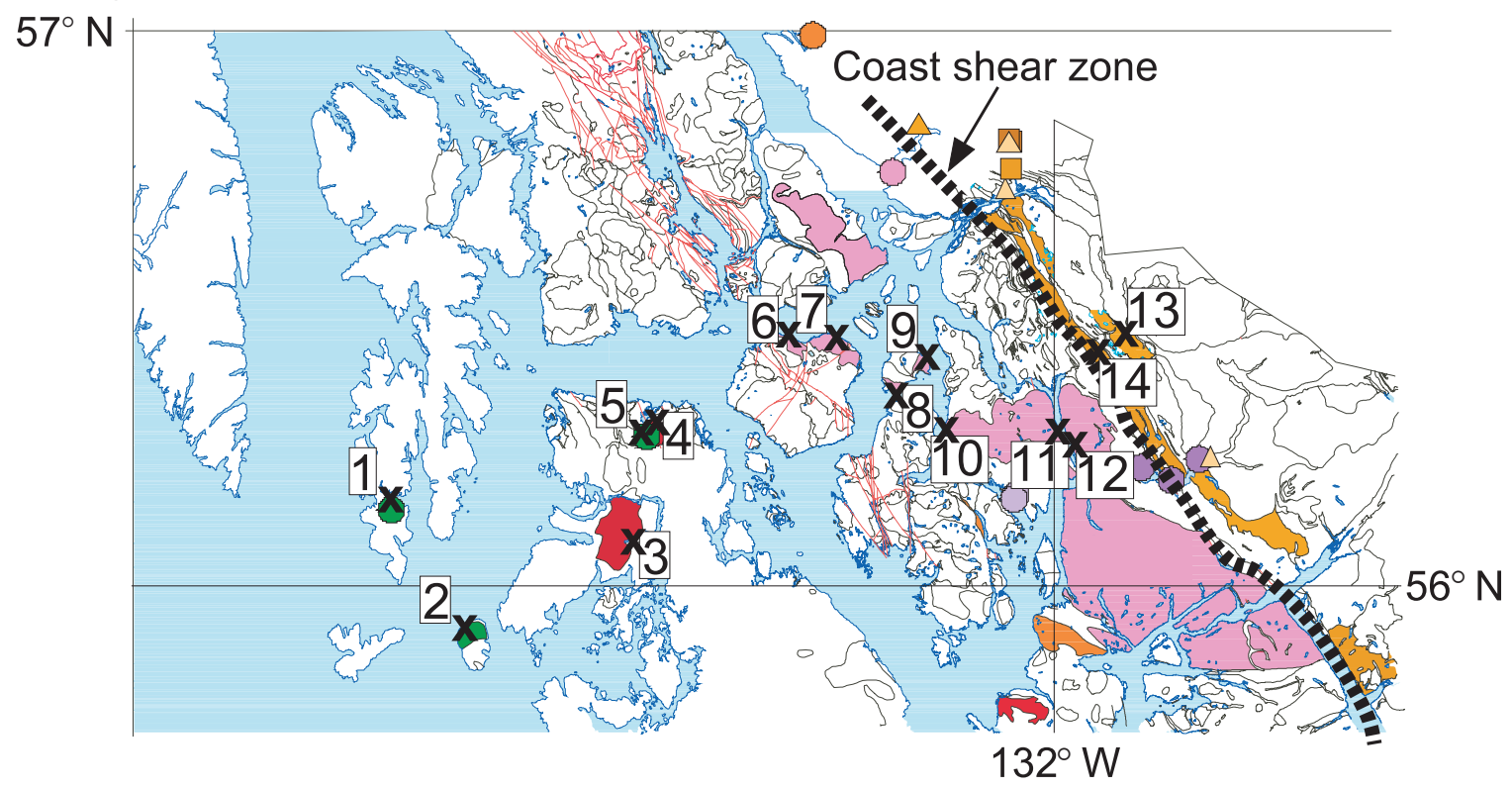

Figure 6. (Continued.) 
A

Coast Plutonic Complex/Paleogene batholith

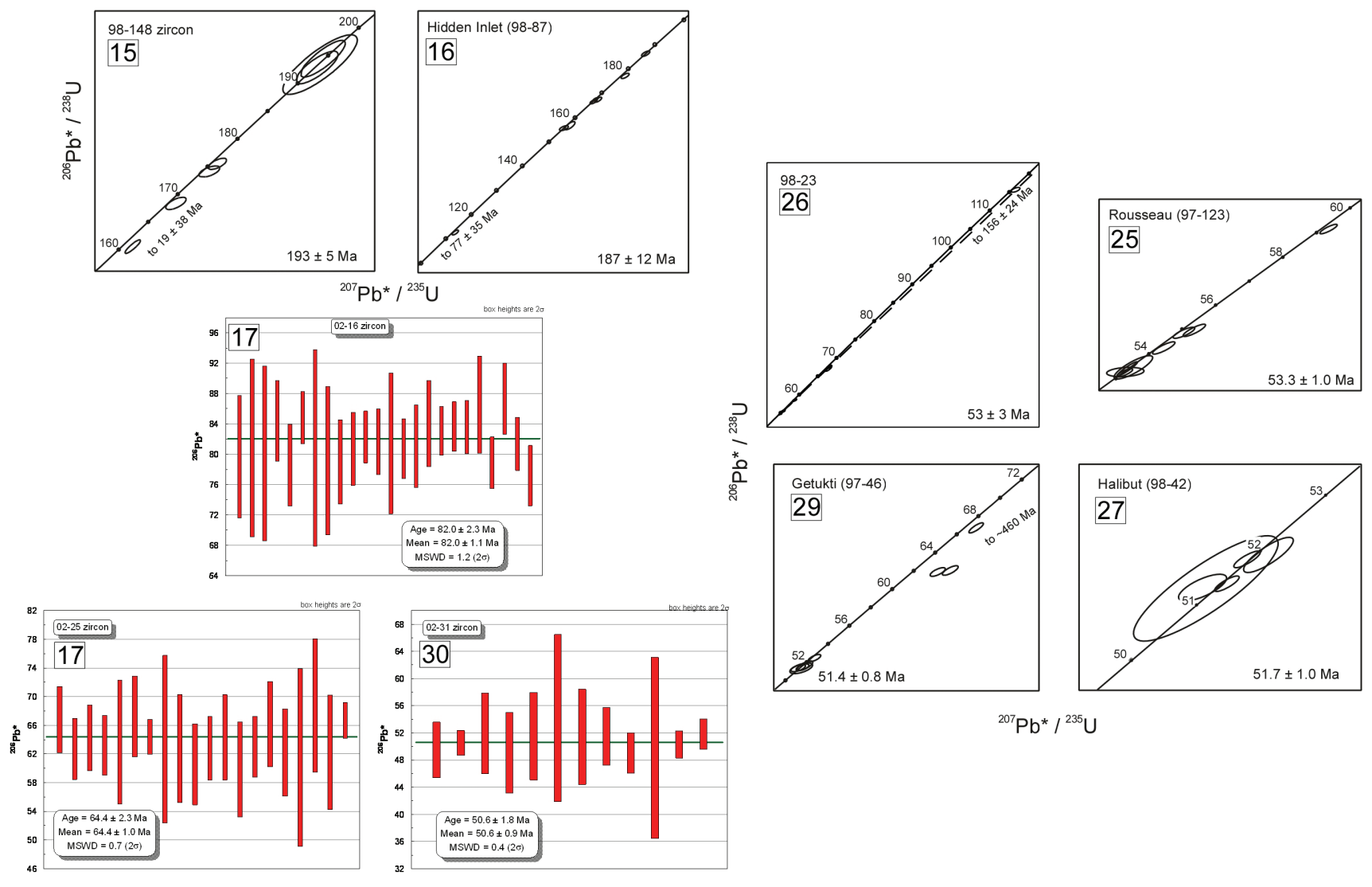

Figure 7. Diagrams of previously unpublished thermal ionization-mass spectrometry (TIMS) and inductively coupled plasma-mass spectrometry (ICP-MS) U-Pb age data for plutons of the Coast Plutonic Complex/Paleogene batholith. (A) Plutons emplaced across the batholith; (B) plutons emplaced along Pearse Canal. The diagram numbers of Figures 7A and 7B correspond to location numbers in Figure 8. MSWD—mean square of weighted deviations. 
B

\section{Coast Plutonic Complex/Pearse Canal}
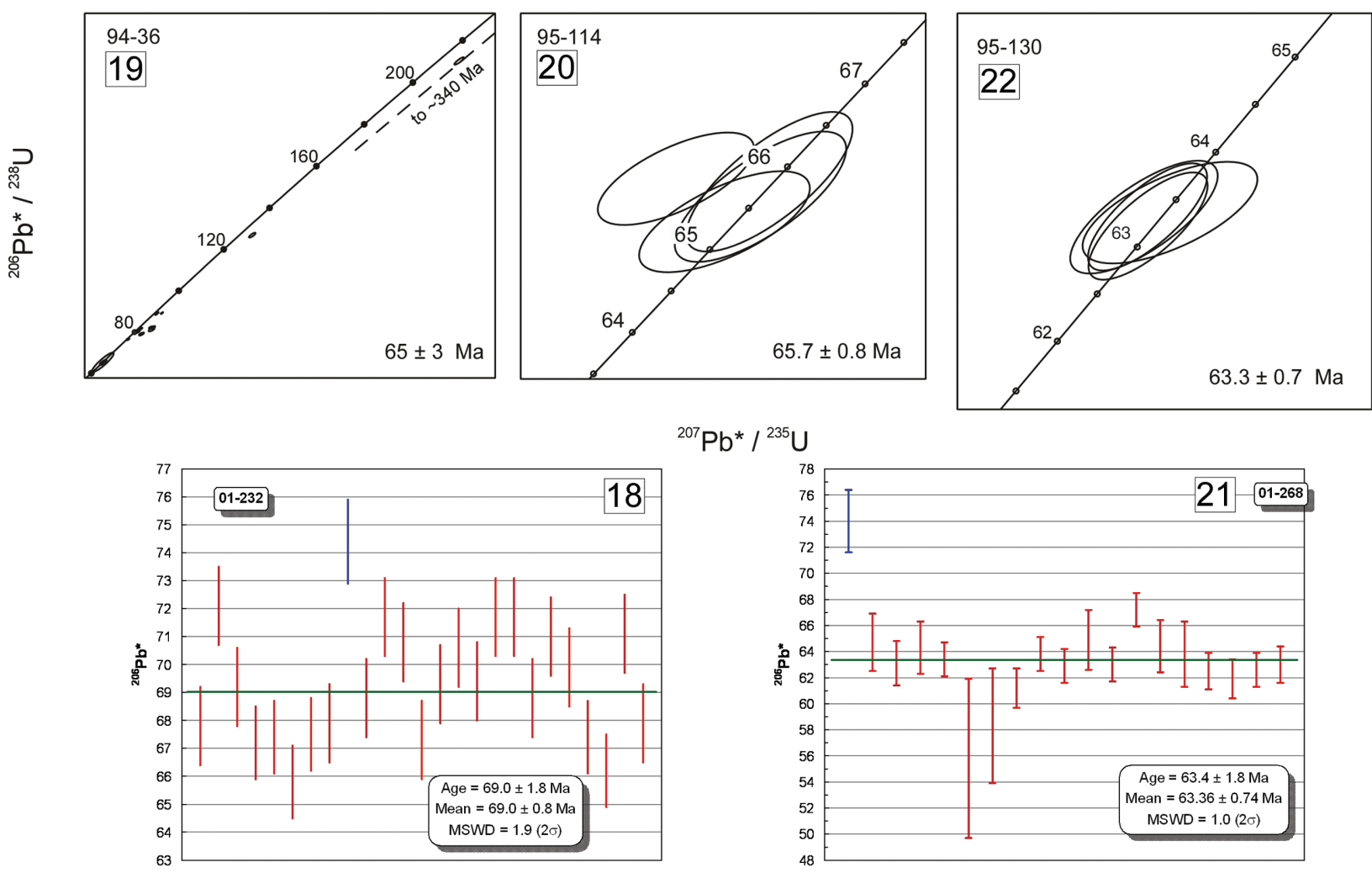

${ }^{207} \mathrm{~Pb}^{*} /{ }^{235} \mathrm{U}$
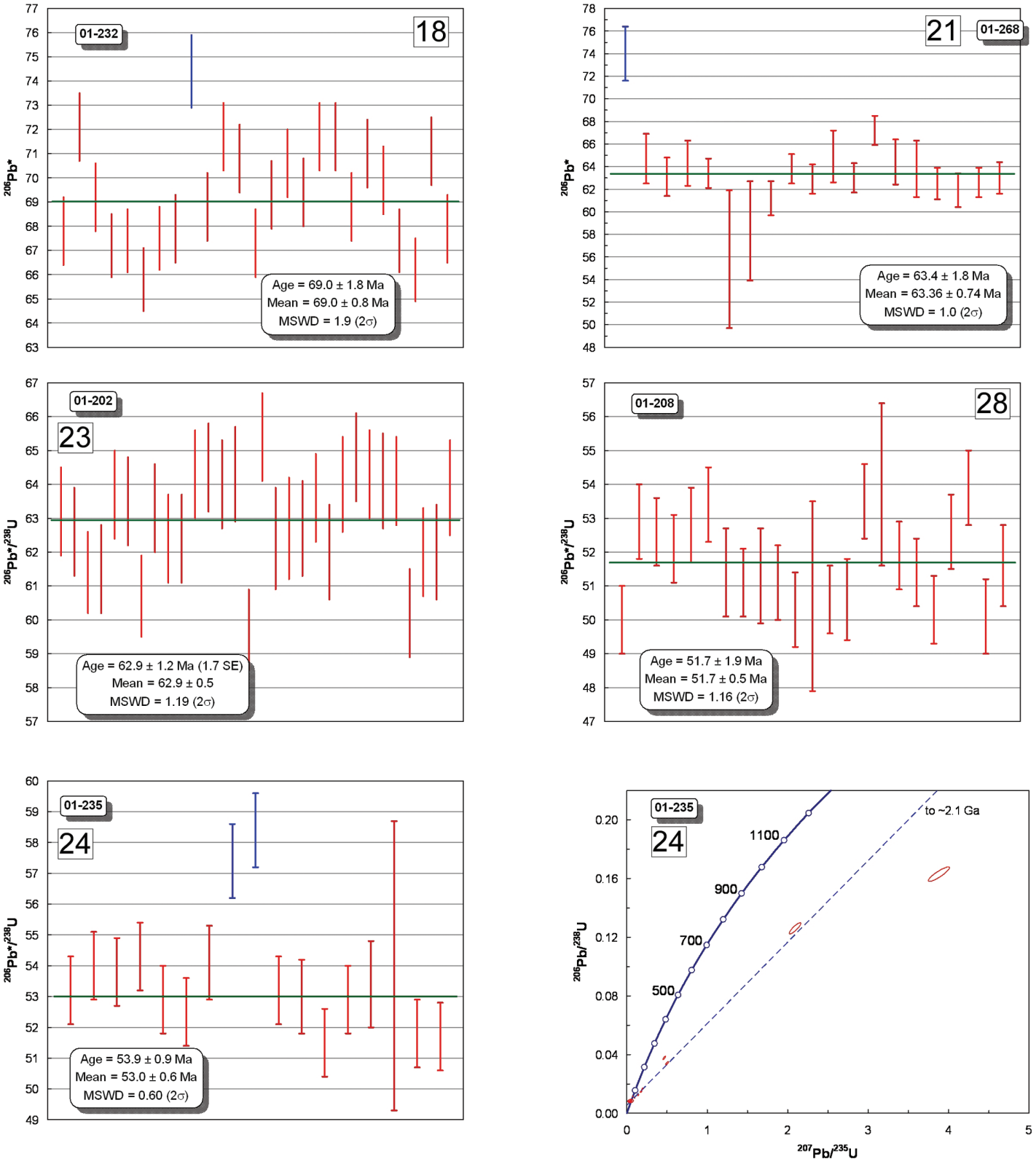

Figure 7. (Continued.)

DR p. 5 


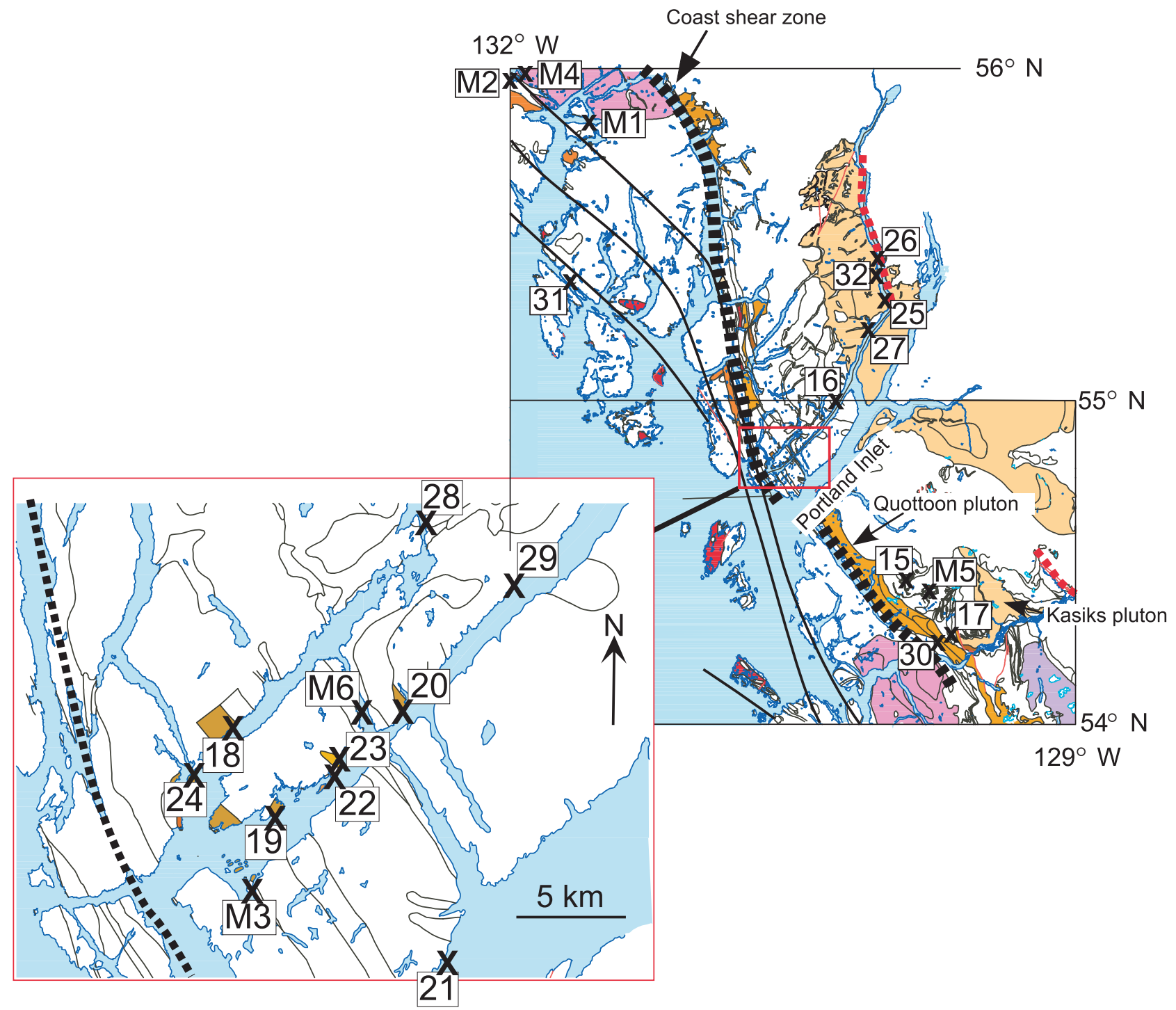

Figure 8. Map showing the locations of dated samples shown in Figures 7 and 11, as well as in Tables 1 and 3-5. The plutons are color keyed to their ages as shown in Figure 5.

Geological Society of America Special Paper 456

Crustal Cross Sections from the Western North American Cordillera

and Elsewhere: Implications for Tectonic and Petrologic Processes

Edited by Robert B. Miller and Arthur W. Snoke

(C) 2009 The Geological Society of America

To accompany Chapter 4, "Mid-Cretaceous-Recent crustal evolution in the central Coast orogen, British Columbia and southeastern Alaska," by Maria Luisa Crawford, Keith A. Klepeis, George E. Gehrels, and Jennifer Lindline 


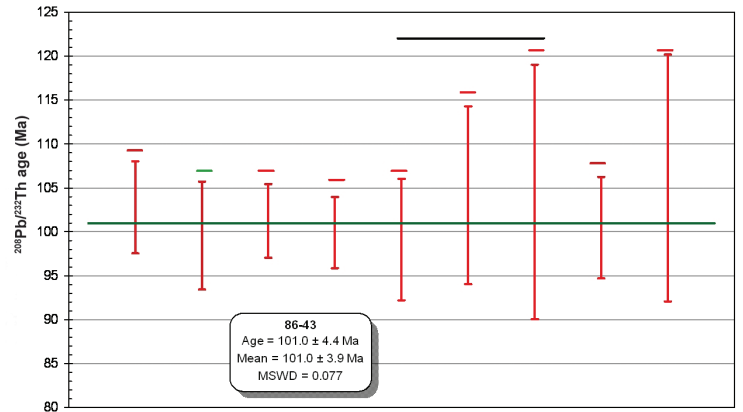

(A)

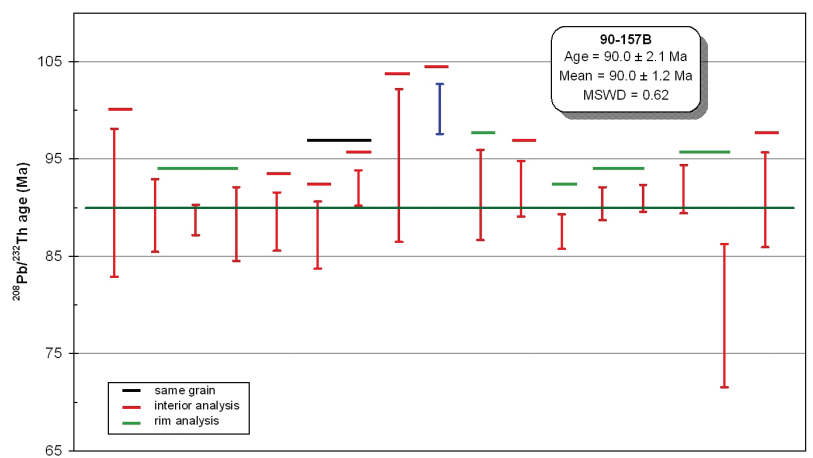

(C)

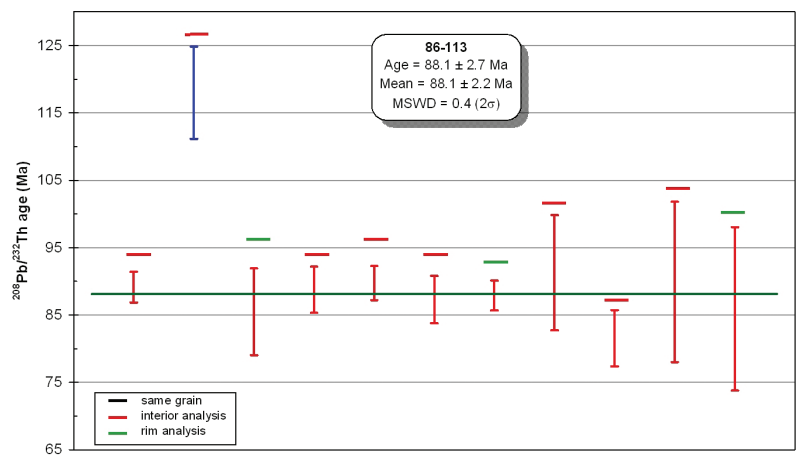

(E)

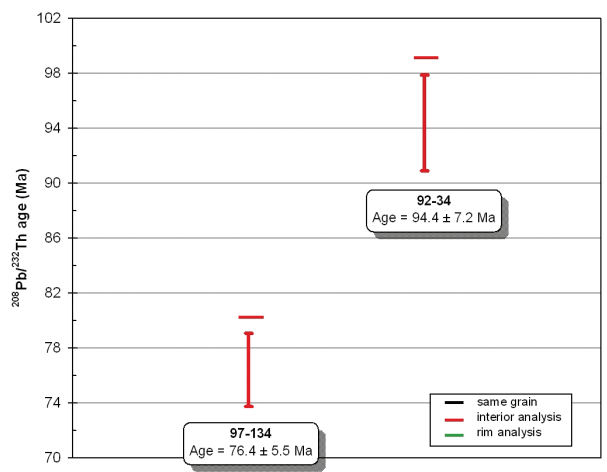

(B)

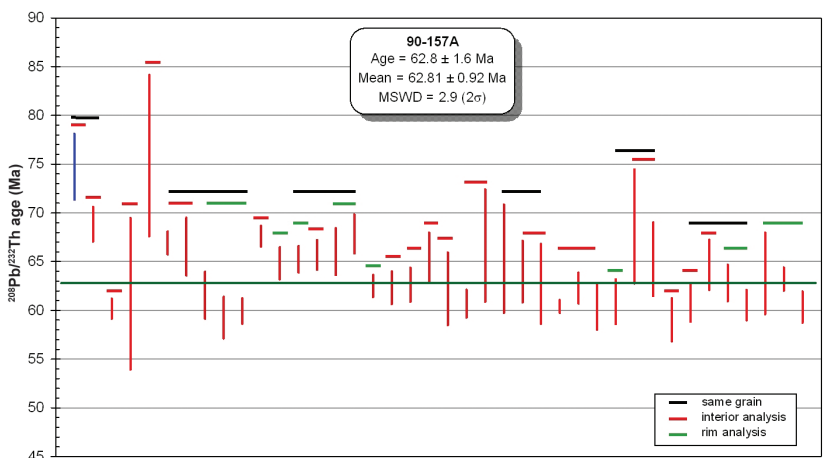

(D)

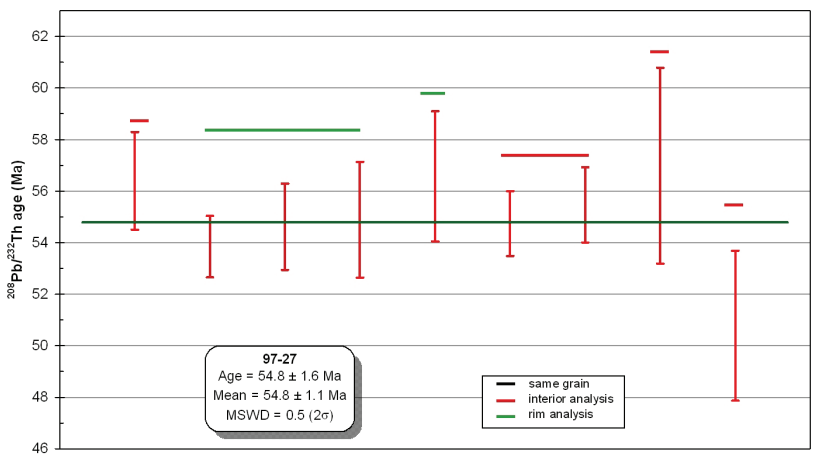

(F)

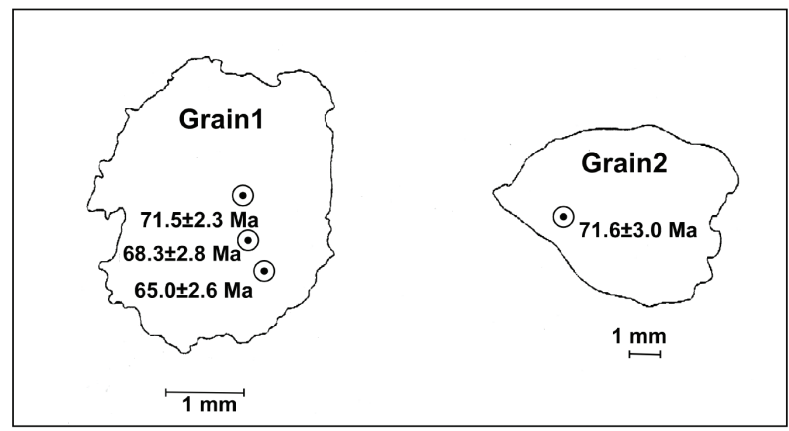

(G)

Figure 11. (A-F) through ICP-MS (inductively coupled plasma-mass spectrometry) monazite ages of metamorphic rocks that host the plutons. The analytical data are presented in Table 3 and summarized in Table 1; the sample locations are in Figure 8. The reported ages are determined from the weighted mean (Ludwig, 2003) of the ${ }^{208} \mathrm{~Pb} /{ }^{32} \mathrm{Th}$ ages. Analyses statistically excluded from the main cluster are shown in blue. Analyses from outer and inner portions of garnet crystals are shown with a green and red bars, respectively. Analyses from the same garnet crystal are indicated with a black bar. Two uncertainties are reported. The smaller uncertainty (labeled "Mean") is based on the scatter and precision of the set of ${ }^{208} \mathrm{~Pb} /{ }^{232} \mathrm{Th}$ ages, weighted according to their measurement errors (shown at $1 \sigma$ ). The larger uncertainty (labeled "Age"), which is the reported uncertainty of the age, is determined as the quadratic sum of the weighted mean error plus the total systematic error for the set of analyses. The systematic error, which includes contributions from the standard calibration, age of the calibration standard, composition_of common Pb, and ${ }^{238} \mathrm{U}$ decay constant, was $2 \%(2 \sigma)$. (G) Line drawing of garnets from Coast Plutonic Complex gneiss collected between the Kasiks and Quottoon plutons showing the locations and ages of dated monazite inclusions. 


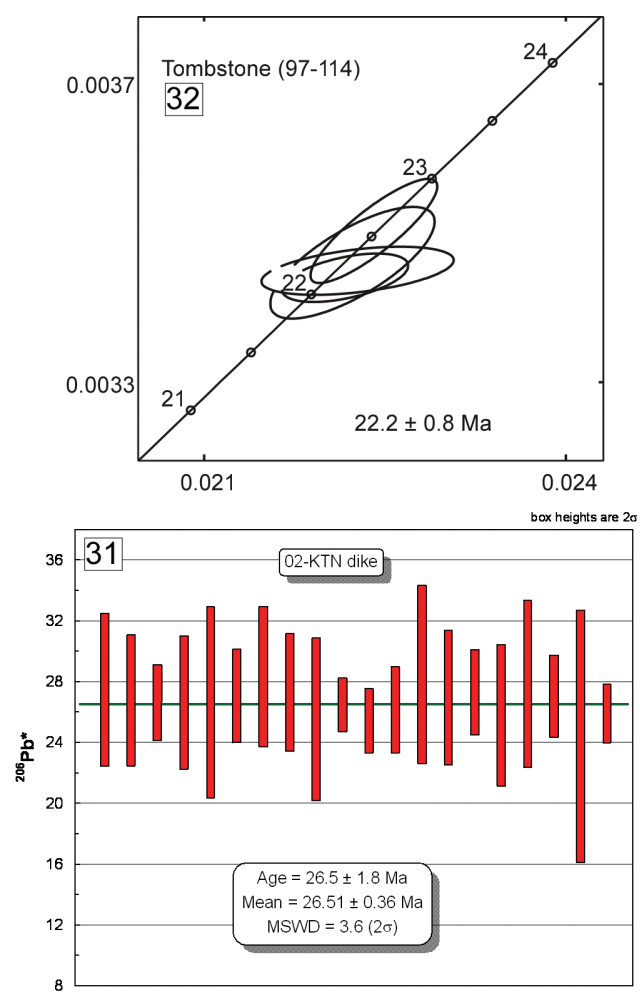

Figure 12. Diagrams of previously unpublished TIMS (thermal ionization-mass spectrometry) and ICP-MS (inductively coupled plasma-mass spectrometry) age data for Miocene plutons. The diagram numbers correspond to location numbers on Figure 8.

Geological Society of America Special Paper 456

Crustal Cross Sections from the Western North American Cordillera and Elsewhere: Implications for Tectonic and Petrologic Processes Edited by Robert B. Miller and Arthur W. Snoke

() 2009 The Geological Society of America

To accompany Chapter 4, "Mid-Cretaceous-Recent crustal evolution in the central Coast orogen, British Columbia and southeastern Alaska," by Maria Luisa Crawford, Keith A. Klepeis, George E. Gehrels, and Jennifer Lindline 
TABLE 2A. WRANGELL TRANSECT: U-Pb (ZIRCON) GEOCHRONOLOGIC ANALYSES BY THERMAL IONIZATION MASS SPECTROMETRY

\begin{tabular}{|c|c|c|c|c|c|c|c|c|c|c|c|}
\hline \multirow[b]{2}{*}{ Sample } & \multirow[b]{2}{*}{$\begin{array}{l}\text { Wt. } \\
(\mu \mathrm{g})\end{array}$} & \multirow[b]{2}{*}{$\begin{array}{l}\mathrm{Pb}_{\mathrm{c}} \\
(\mathrm{pg})\end{array}$} & \multirow[b]{2}{*}{$\begin{array}{c}\mathrm{U} \\
(\mathrm{ppm})\end{array}$} & \multirow[b]{2}{*}{$\frac{{ }^{206} \mathrm{~Pb}}{{ }^{204} \mathrm{~Pb}}$} & \multirow[b]{2}{*}{$\frac{{ }^{206} \mathrm{~Pb}}{{ }^{208} \mathrm{~Pb}}$} & \multicolumn{6}{|c|}{ Apparent ages (Ma) } \\
\hline & & & & & & $\frac{{ }^{206} \mathrm{~Pb}^{\star}}{{ }^{238} \mathrm{U}}$ & \pm & $\frac{{ }^{207} \mathrm{~Pb}^{*}}{{ }^{235} \mathrm{U}}$ & \pm & $\frac{{ }^{207} \mathrm{~Pb}^{\star}}{{ }^{206} \mathrm{~Pb}^{\star}}$ & \pm \\
\hline \multicolumn{12}{|c|}{ Kell Bay Pluton (KP4) } \\
\hline ZB-2 & 28 & 4 & 126 & 1530 & 8.3 & 115.9 & 1.2 & 117 & 1.5 & 139 & 19 \\
\hline ZE-6 & 16 & 3 & 181 & 859 & 6.9 & 113.5 & 1.5 & 113 & 2 & 111 & 31 \\
\hline ZD-3 & 18 & 18 & 407 & 1360 & 7.2 & 114.8 & 1.2 & 114 & 1.8 & 100 & 30 \\
\hline ZA-1 & 21 & 9 & 321 & 788 & 6.6 & 115.7 & 1.4 & 114 & 2.9 & 73 & 51 \\
\hline ZE-4 & 21 & 14 & 363 & 629 & 5.7 & 114.9 & 1.4 & 114 & 2.6 & 95 & 41 \\
\hline \multicolumn{12}{|c|}{ Warren Island (KP48) } \\
\hline ZD-6 & 21 & 4 & 497 & 2740 & 11.2 & 112.2 & 1.0 & 113 & 1.4 & 125 & 16 \\
\hline ZD-5 & 22 & 9 & 618 & 1630 & 9.2 & 110.7 & 1.0 & 110 & 1.9 & 84 & 25 \\
\hline ZD-5 & 20 & 16 & 1219 & 1760 & 9.4 & 112.1 & 0.9 & 111 & 1.8 & 96 & 23 \\
\hline ZE-6 & 23 & 18 & 585 & 865 & 7.8 & 111.1 & 1.1 & 112 & 1.9 & 133 & 30 \\
\hline \multicolumn{12}{|c|}{ El Capitan Pluton (KP26) } \\
\hline ZD-6 & 28 & 3 & 430 & 3640 & 8.8 & 107.9 & 0.9 & 108 & 1.5 & 113 & 22 \\
\hline ZB-2 & 31 & 6 & 357 & 2185 & 7.6 & 108.6 & 1.1 & 109 & 1.7 & 106 & 20 \\
\hline ZE-5 & 18 & 67 & 1398 & 420 & 6.1 & 108.0 & 1.1 & 107 & 3.5 & 83 & 65 \\
\hline ZE-5 & 16 & 9 & 1088 & 2260 & 8.5 & 110.7 & 1.0 & 112 & 2.2 & 140 & 33 \\
\hline ZE-5 & 17 & 10 & 938 & 1710 & 7.9 & 107.9 & 1.3 & 109 & 1.9 & 134 & 23 \\
\hline
\end{tabular}

Grain size: $A=>175 \mu, B=145-175 \mu, C=125-145 \mu, D=100-125 \mu, E=80-100 \mu, F=63-80 \mu, G=45-63 \mu$. $a=a b r a d e d$.

\begin{tabular}{|c|c|c|c|c|c|c|c|c|c|c|c|}
\hline Sample & $\begin{array}{l}\text { Wt. } \\
(\mu \mathrm{g})\end{array}$ & $\begin{array}{l}\mathrm{Pb}_{\mathrm{c}} \\
(\mathrm{pg})\end{array}$ & $\begin{array}{c}U \\
\text { (ppm) }\end{array}$ & $\frac{{ }^{206} \mathrm{~Pb}}{{ }^{204} \mathrm{~Pb}}$ & $\frac{{ }^{206} \mathrm{~Pb}}{{ }^{208} \mathrm{~Pb}}$ & $\frac{{ }^{206} \mathrm{~Pb}^{*}}{{ }^{238} \mathrm{U}}$ & \pm & $\frac{{ }^{207} \mathrm{~Pb}^{*}}{{ }^{235} \mathrm{U}}$ & \pm & $\frac{{ }^{207} \mathrm{~Pb}^{\star}}{{ }^{206} \mathrm{~Pb}^{\star}}$ & \pm \\
\hline \multicolumn{12}{|c|}{ Woronkofski-1 } \\
\hline$\overline{Z G}$ & 9 & & 936 & 1995 & 27.7 & 94.5 & 0.5 & 95.4 & 0.9 & 118 & 15 \\
\hline ZG & 14 & & 1054 & 9800 & 35.7 & 95.5 & 0.5 & 96.2 & 0.7 & 114 & 10 \\
\hline ZG & 10 & & 1760 & 11,700 & 37.7 & 96.8 & 0.5 & 98.6 & 0.6 & 141 & 8 \\
\hline ZF & 160 & & 1089 & 6550 & 27.2 & 102.4 & 0.4 & 108.0 & 0.7 & 233 & 13 \\
\hline ZF & 185 & & 628 & 5040 & 25.4 & 99.3 & 0.5 & 100.9 & 0.8 & 140 & 15 \\
\hline ZC & 132 & & 674 & 4790 & 34.4 & 98.6 & 0.5 & 100.1 & 0.9 & 138 & 16 \\
\hline ZB & 210 & & 518 & 271 & 6.1 & 97.8 & 0.7 & 99.4 & 3.6 & 138 & 78 \\
\hline ZB & 184 & & 322 & 1430 & 19.2 & 99.4 & 0.6 & 103.7 & 1 & 204 & 18 \\
\hline ZA & 41 & & 435 & 1610 & 20.5 & 103.2 & 0.7 & 108.7 & 1.4 & 230 & 23 \\
\hline SA & 42 & & 69 & 83 & 1.9 & 92.5 & 1.7 & 93.1 & 11 & 110 & 240 \\
\hline SA & 49 & & 42 & 79 & 1.8 & 91.1 & 1.8 & 90.6 & 12 & 76 & 260 \\
\hline \multicolumn{12}{|c|}{ Woronkofski-2 } \\
\hline$\overline{Z G}$ & 18 & & 1998 & 10,550 & 39.5 & 88.3 & 0.6 & 88.7 & 0.8 & 98 & 10 \\
\hline ZG & 21 & & 1931 & 11,700 & 39.2 & 89.1 & 0.8 & 90.2 & 0.9 & 118 & 10 \\
\hline ZG & 12 & & 1248 & 8700 & 42.6 & 88.7 & 0.6 & 88.9 & 0.8 & 96 & 11 \\
\hline ZF & 155 & & 1371 & 11,550 & 42.9 & 88.7 & 0.6 & 89.2 & 0.7 & 102 & 8 \\
\hline ZC & 185 & & 1047 & 7600 & 28.9 & 97.2 & 0.5 & 101.7 & 0.7 & 209 & 10 \\
\hline ZB & 205 & & 856 & 1430 & 18.4 & 91.0 & 0.6 & 92.9 & 1.5 & 140 & 30 \\
\hline ZB & 232 & & 475 & 4680 & 24.3 & 104.7 & 0.7 & 114.8 & 1.1 & 329 & 15 \\
\hline SA & 53 & & 201 & 124 & 3.2 & 88.5 & 1.2 & 86.9 & 6 & 46 & 150 \\
\hline SA & 48 & & 129 & 122 & 3.1 & 88.5 & 1.2 & 88.7 & 6.1 & 97 & 150 \\
\hline Grain & l, $B$ & 75 & $25-14$ & 0 & $E=$ & $63 \mu$. & & & & & \\
\hline
\end{tabular}

Notes: All uncertainties are at the $95 \%$ confidence level.

*_radiogenic $\mathrm{Pb}$

Z-zircon, S-Sphene

$\mathrm{Pb}$ - total common $\mathrm{Pb}$ in picograms

${ }^{206} \mathrm{~Pb}_{\mathrm{m}}{ }^{204} \mathrm{~Pb}$ is measured ratio, uncorrected for spike, fractionation, or blank $\mathrm{Pb}$.

${ }^{206} \mathrm{~Pb} /{ }^{208} \mathrm{~Pb}$ is corrected for blank, spike, fractionation, and initial $\mathrm{Pb}$.

$\mathrm{Pb} \& \mathrm{U}$ concentrations have uncertainties of up to $25 \%$ due to uncertainty in grain weight

Constants used: ${ }^{235} \mathrm{U}=9.8485 \times 10^{-10},{ }^{238} \mathrm{U}=1.55125 \times 10^{-10},{ }^{238} \mathrm{U} /{ }^{235} \mathrm{U}=137.88$.

In calculating $U$ concentration and apparent ages, the isotope ratios are adjusted as follows:

(1) Mass dependent corrections factors of: $0.14 \pm 0.06 \% / \mathrm{AMU}$ for $\mathrm{Pb}$ and $0.04 \pm 0.04 \% / \mathrm{AMU}$ for $\mathrm{UO}_{2}$.

(2) $\mathrm{Pb}$ ratios corrected for $0.010 \pm 0.005$ ng blank with ${ }^{206} \mathrm{~Pb} /{ }^{204} \mathrm{~Pb}=18.6 \pm 0.3,{ }^{207} \mathrm{~Pb} /{ }^{204} \mathrm{~Pb}=15.5 \pm 0.3$, and ${ }^{208} \mathrm{~Pb} /{ }^{204} \mathrm{~Pb}=38.0 \pm$ 0.8 .

(3) $U$ has been adjusted for $0.001 \pm 0.001 \mathrm{ng}$ blank.

(4) Initial $\mathrm{Pb}$ composition is from Stacey and Kramers (1975), with uncertainties of 2.0 for ${ }^{206} \mathrm{~Pb} /{ }^{204} \mathrm{~Pb}, 0.3$ for ${ }^{207} \mathrm{~Pb} /{ }^{204} \mathrm{~Pb}$, and 2.0 for ${ }^{208} \mathrm{~Pb} /{ }^{204} \mathrm{~Pb}$.

All analyses conducted using conventional isotope dilution and thermal ionization mass spectrometry, as described by Gehrels (2000).

Isotopic data were processed with programs of Ludwig (1991a, 1991b). 


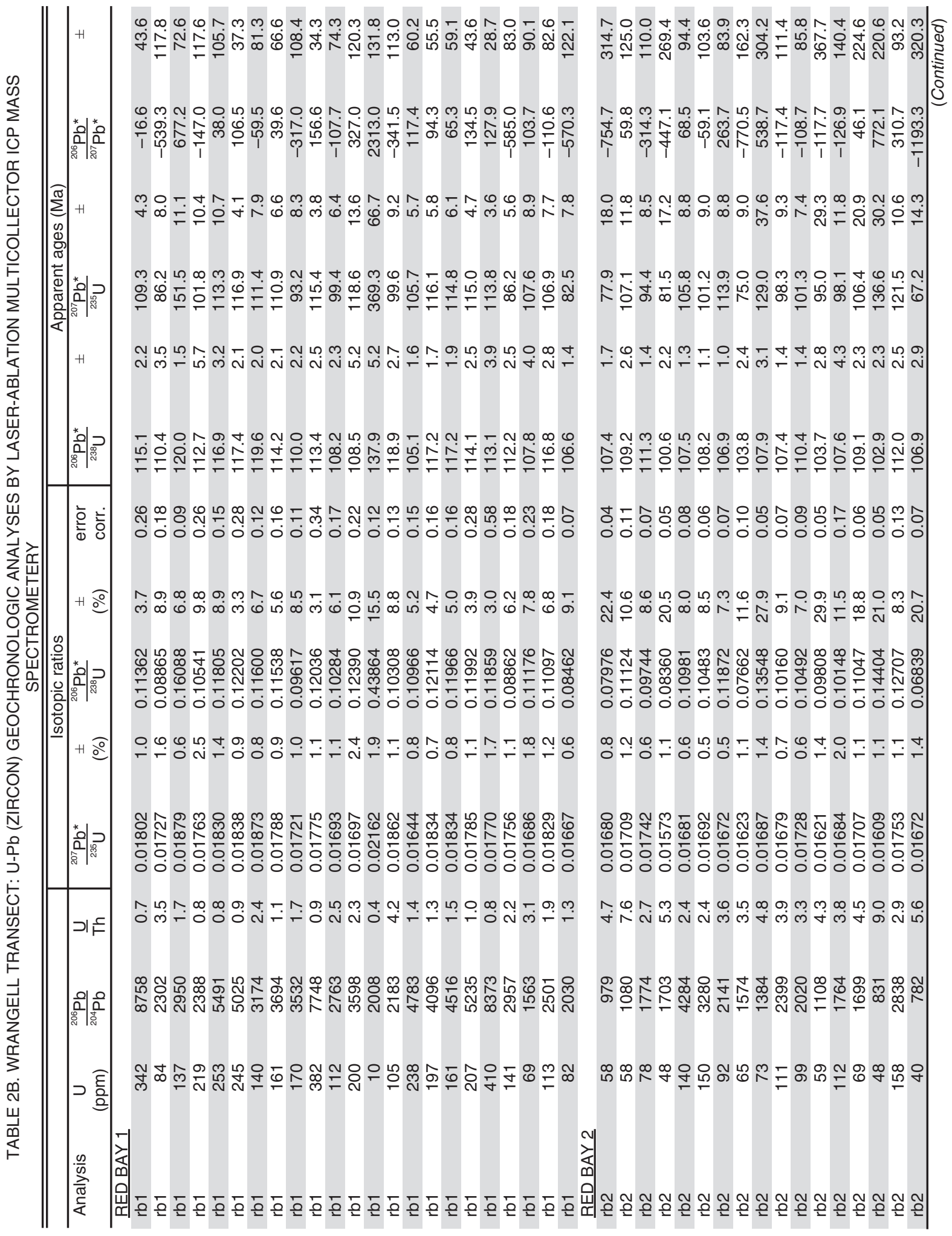




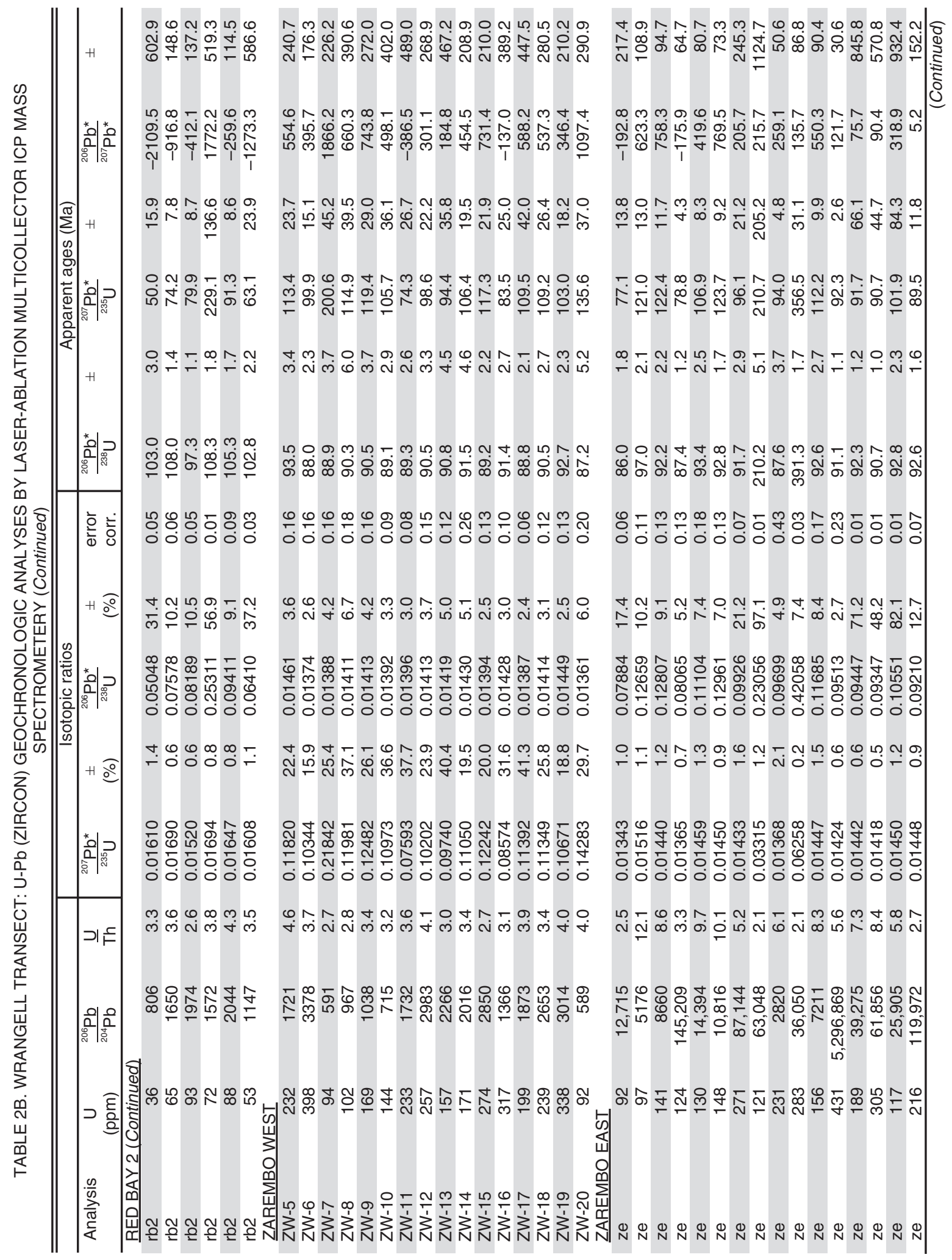




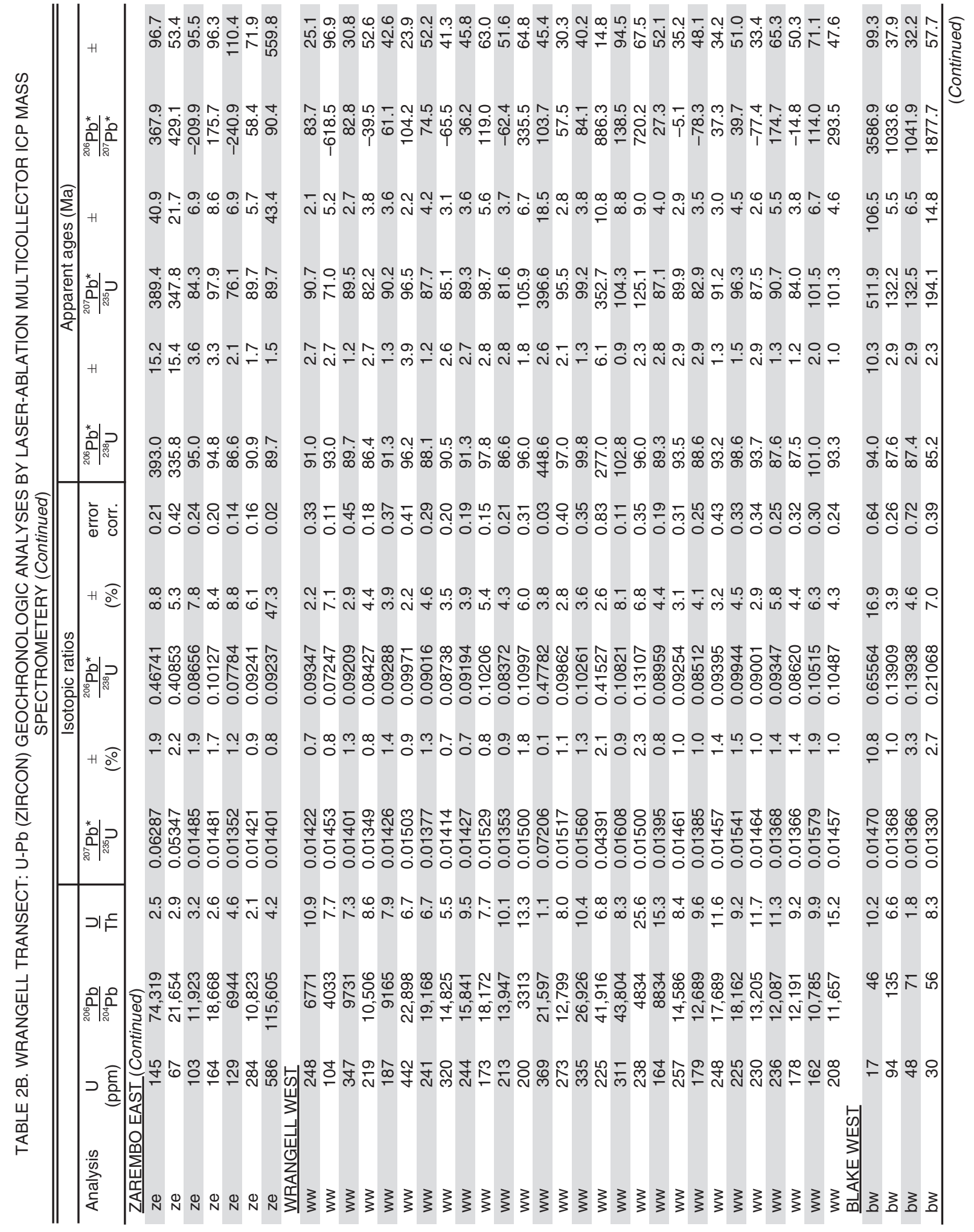




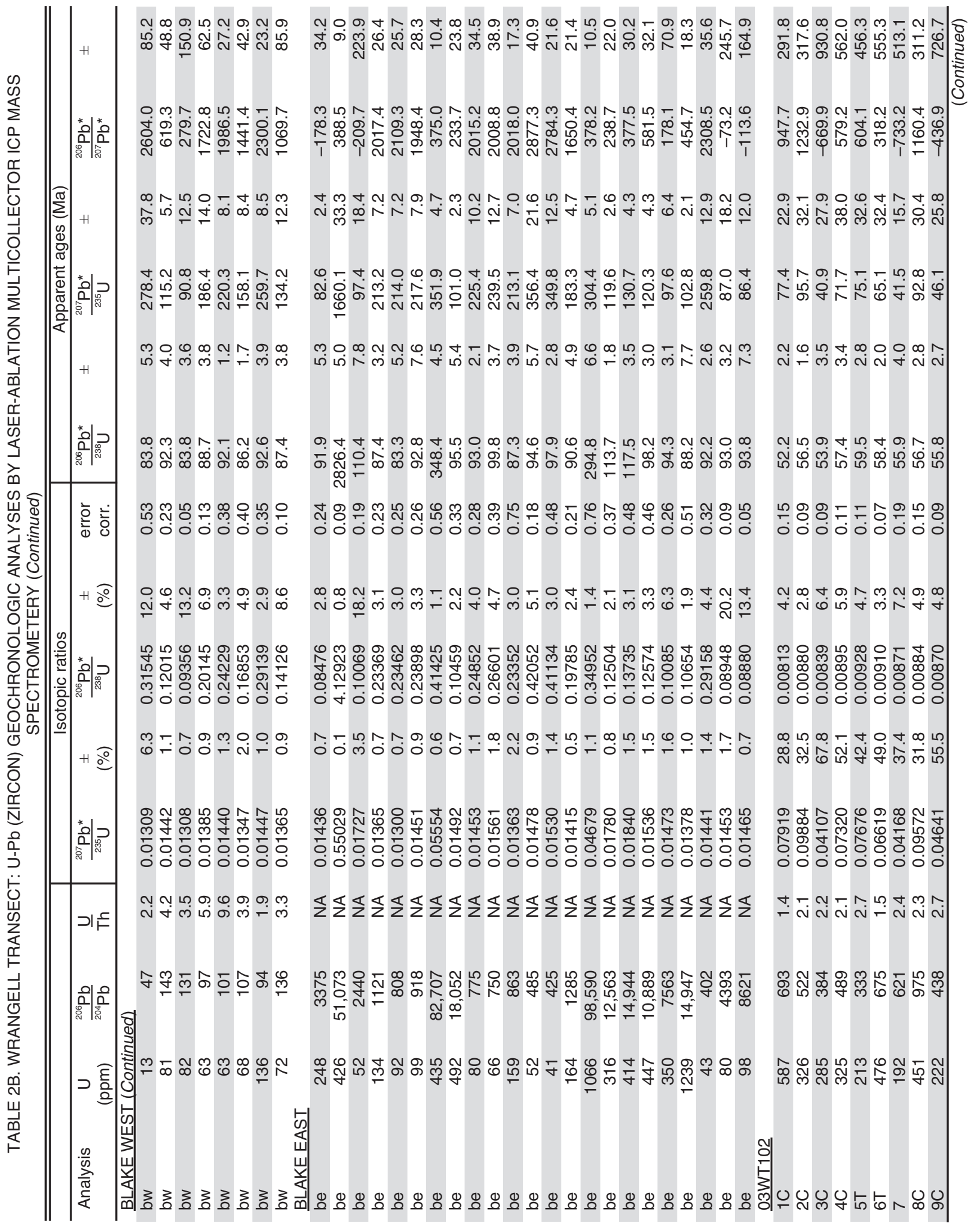




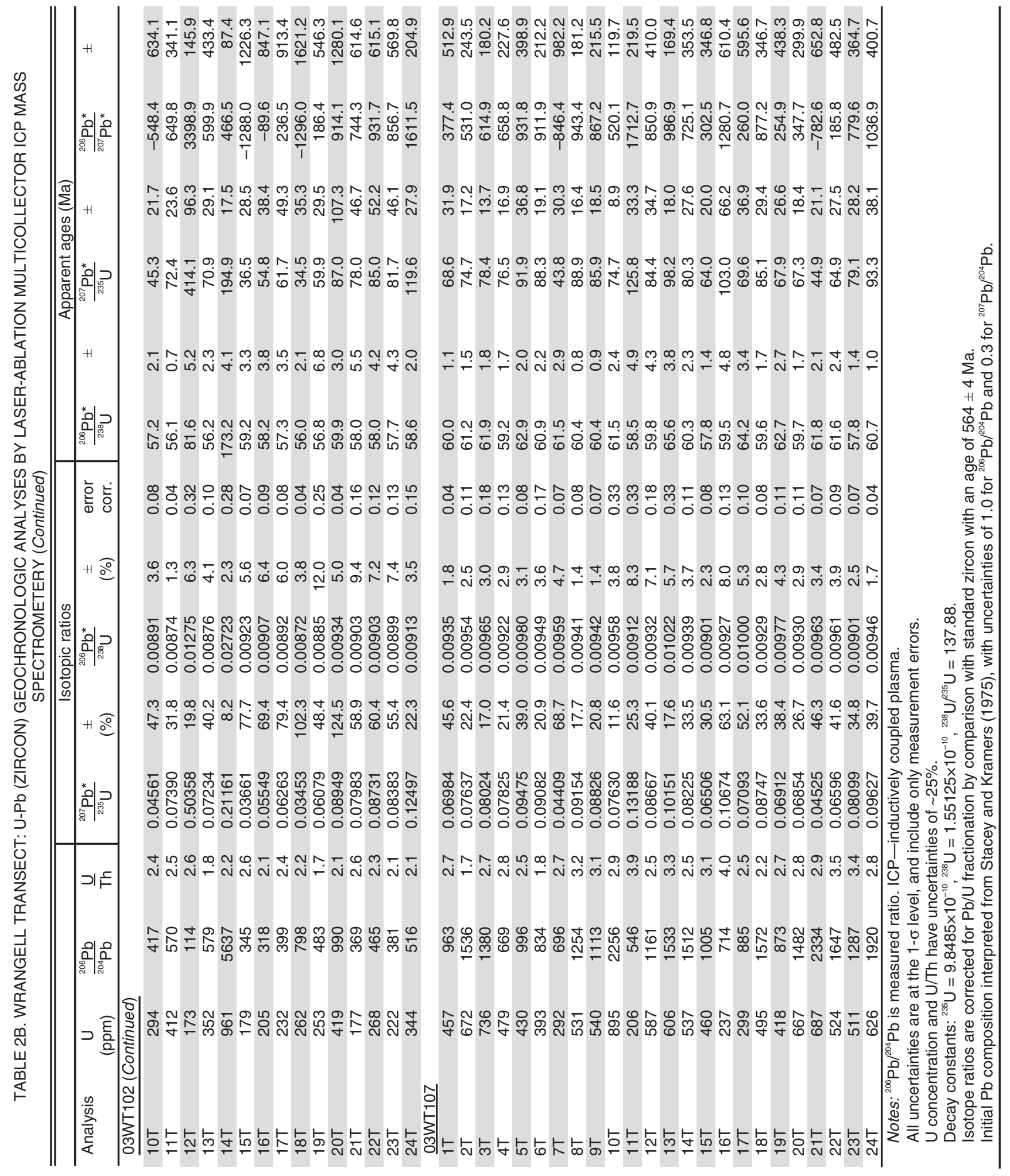




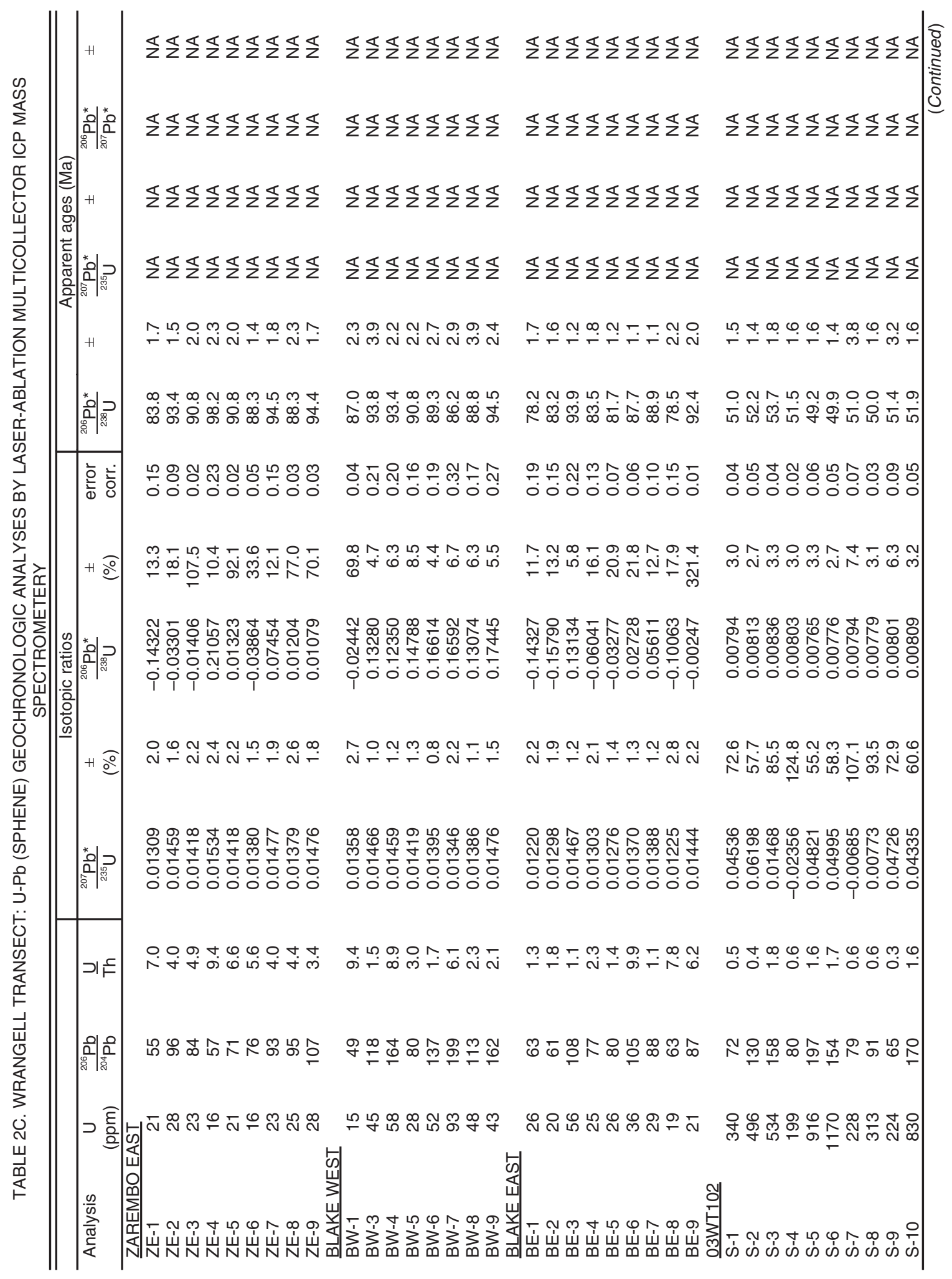




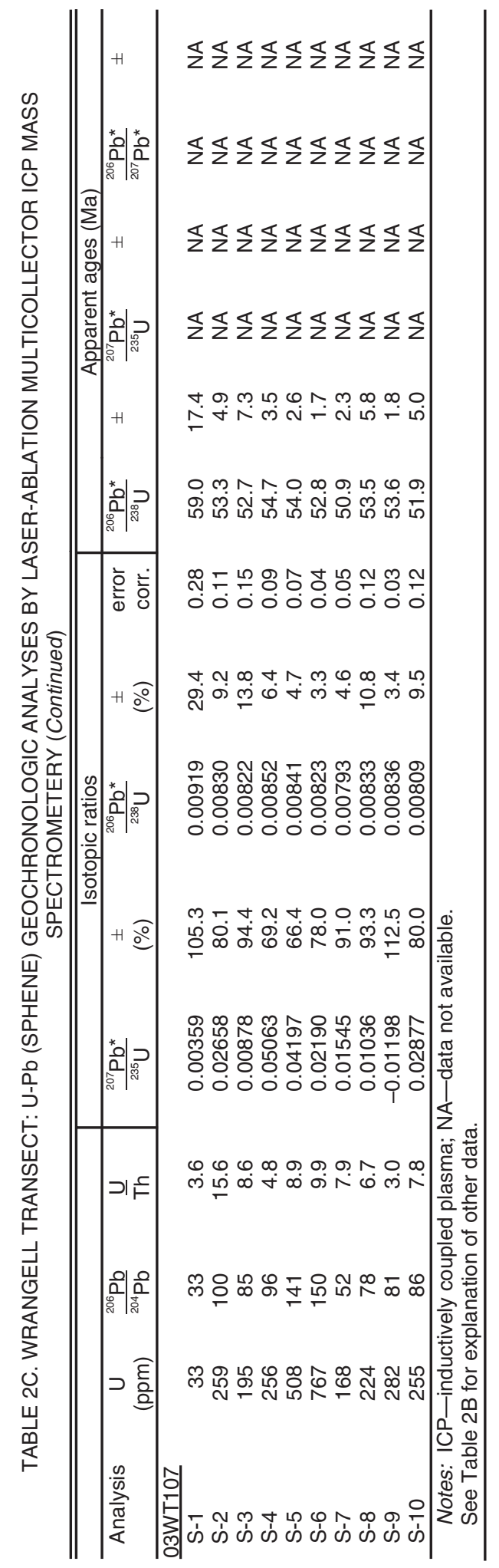

DR p. 16 
TABLE 3. Pb/Th AGES OF MONAZITE INCLUSIONS IN GARNET CRYSTALS

\begin{tabular}{|c|c|c|c|c|c|c|}
\hline Sample & $\begin{array}{c}\text { Th } \\
\text { (ppm) }\end{array}$ & $\begin{array}{ll}{ }^{208} \mathrm{~Pb} \\
{ }^{204} \mathrm{~Pb}\end{array}$ & $\frac{}{{ }^{208} \mathrm{~Pb}}$ & $\begin{array}{c} \pm \\
(\%)\end{array}$ & $\begin{array}{ll}{ }^{208} \mathrm{~Pb} & \begin{array}{l}\text { Age } \\
{ }^{232} \mathrm{Th} \\
(\mathrm{Ma})\end{array}\end{array}$ & $\begin{array}{c} \pm \\
(\mathrm{Ma})\end{array}$ \\
\hline $97-134-1 \mathrm{~A}$ & 1323 & 454 & 0.00379 & 3.5 & 76.4 & 2.7 \\
\hline$\underline{92-34-1}$ & 839 & 800 & 0.00468 & 3.7 & 94.4 & 3.5 \\
\hline $97-27-2$ & 1383 & 712 & 0.00279 & 3.4 & 56.4 & 1.9 \\
\hline$-3 A$ & 4424 & 469 & 0.00267 & 2.2 & 53.8 & 1.2 \\
\hline$-3 B$ & 4020 & 459 & 0.00271 & 3.1 & 54.6 & 1.7 \\
\hline$-3 C$ & 4045 & 468 & 0.00272 & 4.1 & 54.9 & 2.2 \\
\hline-4 & 2930 & 640 & 0.00280 & 4.5 & 56.6 & 2.5 \\
\hline$-9 A$ & 4300 & 439 & 0.00271 & 2.3 & 54.7 & 1.3 \\
\hline$-9 B$ & 4898 & 490 & 0.00275 & 2.6 & 55.5 & 1.5 \\
\hline-6 & 2477 & 378 & 0.00282 & 6.7 & 57.0 & 3.8 \\
\hline-7 & 281 & 306 & 0.00252 & 5.7 & 50.8 & 2.9 \\
\hline $90-157 \mathrm{~B}-1$ & 67 & 687 & 0.00449 & 8.4 & 90.5 & 7.6 \\
\hline-2 & 2169 & 886 & 0.00442 & 4.2 & 89.2 & 3.7 \\
\hline$-2 B$ & 3969 & 762 & 0.00440 & 1.8 & 88.7 & 1.6 \\
\hline$-2 C$ & 2122 & 862 & 0.00438 & 4.3 & 88.3 & 3.8 \\
\hline-3 & 1828 & 754 & 0.00439 & 3.4 & 88.6 & 3.0 \\
\hline-4 & 1179 & 568 & 0.00432 & 4.0 & 87.2 & 3.5 \\
\hline-5 & 3333 & 658 & 0.00456 & 2.0 & 92.0 & 1.8 \\
\hline-6 & 1687 & 696 & 0.00468 & 8.3 & 94.3 & 7.9 \\
\hline-7 & 3024 & 657 & 0.00497 & 2.6 & 100.1 & 2.6 \\
\hline-8 & 1195 & 423 & 0.00453 & 5.1 & 91.3 & 4.6 \\
\hline-9 & 3931 & 1146 & 0.00456 & 3.1 & 91.9 & 2.9 \\
\hline-10 & 3659 & 751 & 0.00434 & 2.0 & 87.5 & 1.8 \\
\hline$-11 A$ & 3638 & 562 & 0.00448 & 1.9 & 90.4 & 1.7 \\
\hline$-11 B$ & 4747 & 643 & 0.00451 & 1.5 & 91.0 & 1.4 \\
\hline-12 & 3655 & 488 & 0.00456 & 2.7 & 91.9 & 2.5 \\
\hline$-12 B$ & 209 & 285 & 0.00391 & 9.3 & 78.9 & 7.4 \\
\hline-13 & 1951 & 457 & 0.00450 & 5.4 & 90.8 & 4.9 \\
\hline $90-157 A-1$ & 1239 & 493 & 0.00370 & 4.5 & 74.7 & 3.4 \\
\hline-2 & 1791 & 476 & 0.00341 & 2.6 & 68.8 & 1.8 \\
\hline-3 & 4894 & 621 & 0.00298 & 1.7 & 60.2 & 1.0 \\
\hline-5 & 858 & 759 & 0.00306 & 12.6 & 61.7 & 7.8 \\
\hline-6 & 364 & 580 & 0.00376 & 10.9 & 75.9 & 8.3 \\
\hline$-10 A$ & 4126 & 551 & 0.00332 & 1.8 & 66.9 & 1.2 \\
\hline$-10 B$ & 2685 & 497 & 0.00330 & 4.4 & 66.5 & 3.0 \\
\hline$-11 A$ & 2055 & 224 & 0.00305 & 3.9 & 61.5 & 2.4 \\
\hline$-11 B$ & 2008 & 411 & 0.00294 & 3.6 & 59.3 & 2.1 \\
\hline$-11 C$ & 4403 & 420 & 0.00297 & 2.2 & 59.9 & 1.3 \\
\hline-12 & 3815 & 567 & 0.00335 & 1.6 & 67.6 & 1.1 \\
\hline-13 & 594 & 545 & 0.00321 & 2.5 & 64.8 & 1.6 \\
\hline-14 & 5310 & 495 & 0.00323 & 2.1 & 65.2 & 1.4 \\
\hline-15 & 6827 & 810 & 0.00326 & 2.3 & 65.7 & 1.5 \\
\hline$-16 \mathrm{~A}$ & 3075 & 353 & 0.00327 & 3.6 & 66.0 & 2.4 \\
\hline$-16 \mathrm{~B}$ & 3219 & 347 & 0.00336 & 2.9 & 67.8 & 2.0 \\
\hline-17 & 3702 & 477 & 0.00310 & 1.8 & 62.5 & 1.1 \\
\hline$-18 \mathrm{~A}$ & 2279 & 294 & 0.00309 & 2.7 & 62.3 & 1.7 \\
\hline$-18 B$ & 3072 & 425 & 0.00310 & 2.8 & 62.6 & 1.8 \\
\hline-19 & 1905 & 620 & 0.00324 & 4.0 & 65.4 & 2.6 \\
\hline-20 & 1453 & 529 & 0.00308 & 6.0 & 62.2 & 3.7 \\
\hline$-21 A$ & 2444 & 474 & 0.00301 & 2.4 & 60.7 & 1.4 \\
\hline$-21 B$ & 3513 & 427 & 0.00330 & 8.6 & 66.6 & 5.8 \\
\hline-24 & 1575 & 512 & 0.00324 & 8.5 & 65.3 & 5.6 \\
\hline$-25 A$ & 2405 & 782 & 0.00317 & 4.9 & 64.0 & 3.2 \\
\hline$-25 B$ & 3234 & 794 & 0.00311 & 6.5 & 62.7 & 4.1 \\
\hline$-26 A$ & 4074 & 467 & 0.00299 & 1.1 & 60.4 & 0.7 \\
\hline$-26 B$ & 3321 & 362 & 0.00309 & 2.5 & 62.3 & 1.6 \\
\hline$-26 C$ & 3083 & 396 & 0.00299 & 3.8 & 60.3 & 2.3 \\
\hline-27 & 3023 & 494 & 0.00302 & 3.8 & 60.9 & 2.3 \\
\hline$-28 A$ & 1129 & 551 & 0.00340 & 8.5 & 68.6 & 5.9 \\
\hline$-28 B$ & 759 & 555 & 0.00323 & 5.8 & 65.2 & 3.8 \\
\hline-29 & 1534 & 394 & 0.00292 & 3.7 & 59.0 & 2.2 \\
\hline
\end{tabular}


TABLE 3. Pb/Th AGES OF MONAZITE INCLUSIONS IN GARNET CRYSTALS (Continued)

\begin{tabular}{|c|c|c|c|c|c|c|}
\hline Sample & $\begin{array}{c}\text { Th } \\
\text { (ppm) }\end{array}$ & $\frac{{ }^{208} \mathrm{~Pb}}{{ }^{204} \mathrm{~Pb}}$ & $\frac{{ }^{208} \mathrm{~Pb}}{{ }^{232} \mathrm{Th}}$ & $\begin{array}{c} \pm \\
\%)\end{array}$ & $\begin{array}{l}{ }^{208} \mathrm{~Pb} \text { Age } \\
{ }^{232} \mathrm{Th}(\mathrm{Ma})\end{array}$ & $\begin{array}{c} \pm \\
(\mathrm{Ma})\end{array}$ \\
\hline-30 & 2550 & 227 & 0.00301 & 3.2 & 60.8 & 2.0 \\
\hline-31 & 1687 & 268 & 0.00320 & 4.0 & 64.7 & 2.6 \\
\hline$-32 A$ & 4685 & 461 & 0.00311 & 2.9 & 62.8 & 1.9 \\
\hline$-32 B$ & 4291 & 373 & 0.00300 & 2.6 & 60.5 & 1.6 \\
\hline$-33 A$ & 4149 & 556 & 0.00316 & 6.6 & 63.8 & 4.2 \\
\hline$-33 B$ & 6463 & 580 & 0.00313 & 1.9 & 63.2 & 1.2 \\
\hline$-33 C$ & 6840 & 557 & 0.00299 & 2.7 & 60.4 & 1.6 \\
\hline 86-113-1 & 2331 & 490 & 0.00442 & 2.5 & 89.1 & 2.3 \\
\hline-2 & 184 & 960 & 0.00585 & 5.8 & 118.0 & 6.8 \\
\hline-6 & 1161 & 376 & 0.00424 & 7.5 & 85.5 & 6.4 \\
\hline-11 & 93 & 420 & 0.00440 & 3.8 & 88.7 & 3.4 \\
\hline-14 & 409 & 590 & 0.00445 & 2.8 & 89.7 & 2.5 \\
\hline-16 & 808 & 520 & 0.00433 & 4.0 & 87.3 & 3.5 \\
\hline-18 & 618 & 522 & 0.00436 & 2.5 & 87.9 & 2.2 \\
\hline-21 & 244 & 480 & 0.00453 & 9.4 & 91.3 & 8.6 \\
\hline-23 & 1532 & 616 & 0.00404 & 5.1 & 81.6 & 4.2 \\
\hline-24 & 167 & 331 & 0.00446 & 13.2 & 89.9 & 11.9 \\
\hline-25 & 1561 & 568 & 0.00426 & 14.1 & 85.9 & 12.1 \\
\hline \multicolumn{7}{|c|}{$\begin{array}{l}\text { Notes: All uncertainties are reported at the } 1 \text {-sigma level, and include only measurement errors } \\
\mathrm{U} \text { concentration and } \mathrm{U} / \mathrm{Th} \text { are calibrated relative to NIST SRM } 610 \text { and are accurate to } \sim 20 \% \text {. } \\
\text { Common } \mathrm{Pb} \text { correction is from }{ }^{204} \mathrm{~Pb} \text {, with composition interpreted from Stacey and Kramers } \\
\text { (1975) and uncertainties of } 2.0 \text { for }{ }^{208} \mathrm{~Pb}{ }^{204} \mathrm{~Pb} \text {. }{ }^{208} \mathrm{~Pb} /{ }^{232} \text { Th fractionation is calibrated relative to } \\
\text { standard monazite } 44069 \text { (Aleinikoff et al., 2006). Th decay constant for }{ }^{232} \mathrm{Th}=4.9475 \mathrm{E}-11 \text {. }\end{array}$} \\
\hline
\end{tabular}


2009156

TABLE 4A. PALEOGENE BATHOLITH. U-Pb (ZIRCON) GEOCHRONOLOGIC ANALYSES BY THERMAL IONIZATION MASS SPECTROMETRY

\begin{tabular}{|c|c|c|c|c|c|c|c|c|c|c|c|}
\hline \multirow[b]{2}{*}{ Sample } & \multirow[b]{2}{*}{$\begin{array}{l}\text { Wt. } \\
(\mu \mathrm{g})\end{array}$} & \multirow[b]{2}{*}{$\begin{array}{l}\mathrm{Pb}_{\mathrm{c}} \\
(\mathrm{pg})\end{array}$} & \multirow[b]{2}{*}{$\begin{array}{c}\mathrm{U} \\
(\mathrm{ppm})\end{array}$} & \multirow[b]{2}{*}{$\frac{{ }^{206} \mathrm{~Pb}}{{ }^{204} \mathrm{~Pb}}$} & \multirow[b]{2}{*}{$\frac{{ }^{206} \mathrm{~Pb}}{{ }^{208} \mathrm{~Pb}}$} & \multicolumn{6}{|c|}{ Apparent ages (Ma) } \\
\hline & & & & & & $\frac{{ }^{206} \mathrm{~Pb}^{*}}{{ }^{238} \mathrm{U}}$ & \pm & $\frac{{ }^{207} \mathrm{~Pb}^{*}}{{ }^{235} \mathrm{U}}$ & \pm & $\frac{{ }^{207} \mathrm{~Pb}^{*}}{{ }^{206} \mathrm{~Pb}^{*}}$ & \pm \\
\hline \multicolumn{12}{|l|}{$98-148$} \\
\hline ZAa-1 & 21 & 1 & 169 & 1280 & 13 & 194 & 1 & 194 & 1.7 & 191 & 21 \\
\hline ZAa-1 & 19 & 2 & 209 & 1085 & 10 & 192.8 & 1 & 193 & 1.6 & 196 & 23 \\
\hline ZAa-1 & 24 & 1 & 88 & 670 & 11 & 193.4 & 2 & 194 & 2.8 & 197 & 37 \\
\hline ZA-6 & 180 & 35 & 413 & 3250 & 15 & 175.5 & 1 & 177 & 0.7 & 192 & 14 \\
\hline ZB-15 & 165 & 23 & 432 & 4409 & 17 & 174.1 & 1 & 175 & 0.9 & 191 & 17 \\
\hline ZB-15 & 152 & 25 & 557 & 4702 & 15 & 168.3 & 1 & 170 & 0.9 & 189 & 16 \\
\hline $\begin{array}{l}\text { ZE-30 } \\
\text { Hidden }\end{array}$ & \multicolumn{8}{|c|}{ Hidden (98-87) } & 0.7 & 189 & 8 \\
\hline $\mathrm{ZAa}-1$ & 32 & 3 & 691 & 7560 & 9 & 186.2 & 1 & 187 & 0.7 & 189 & 12 \\
\hline ZAa-1 & 34 & 8 & 641 & 2994 & 10 & 176.9 & 1 & 179 & 0.8 & 206 & 14 \\
\hline ZAa-1 & 24 & 5 & 321 & 2296 & 12 & 156.6 & 1 & 158 & 1.2 & 180 & 18 \\
\hline ZAa-1 & 27 & 14 & 1133 & 2708 & 8 & 167.5 & 1 & 169 & 0.9 & 182 & 16 \\
\hline $\mathrm{ZA}-1$ & 29 & 5 & 1049 & 4997 & 11 & 166.5 & 1 & 167 & 0.7 & 173 & 10 \\
\hline$Z A-1$ & 45 & 16 & 386 & 1275 & 10 & 155.6 & 1 & 156 & 1.1 & 161 & 20 \\
\hline ZA-1 & 41 & 13 & 1445 & 3633 & 14 & 113 & 1 & 114 & 0.8 & 136 & 12 \\
\hline 94-36 & & & & & & & & & & & \\
\hline ZA-1 & 15 & 18 & 1250 & 1505 & 6 & 65.2 & 1 & 65 & 1.3 & 74 & 29 \\
\hline ZA-1 & 14 & 11 & 391 & 43,800 & 13 & 81.4 & 1 & 83 & 0.8 & 113 & 16 \\
\hline ZA-1 & 20 & 15 & 1473 & 15,500 & 13 & 88.8 & 1 & 90 & 0.7 & 121 & 11 \\
\hline ZA-1 & 18 & 9 & 967 & 12,600 & 16 & 79 & 1 & 83 & 0.6 & 208 & 10 \\
\hline ZA-1 & 14 & 17 & 1085 & 2820 & 6 & 126.6 & 1 & 132 & 1.2 & 234 & 12 \\
\hline ZA-1 & 12 & 38 & 650 & 2900 & 16.5 & 82.09 & 0.5 & 88.3 & 0.6 & 258.0 & 13 \\
\hline ZA-1 & 17 & 24 & 1481 & 13,300 & 5.6 & 209.9 & 1.5 & 218.3 & 1.7 & 310.0 & 8 \\
\hline ZB-10 & 180 & 32 & 741 & 4780 & 12.8 & 80 & 0.4 & 81.5 & 0.6 & 124.0 & 13 \\
\hline ZB-10 & 165 & 20 & 695 & 14,280 & 11.7 & 89.1 & 0.4 & 92.3 & 0.5 & 177.0 & 18 \\
\hline ZD-20 & 140 & 19 & 276 & 3190 & 12.1 & 78.9 & 0.6 & 82.9 & 0.9 & 201.0 & 18 \\
\hline ZE-30 & 168 & 16 & 275 & 6190 & 13.1 & 82.1 & 0.5 & 87.4 & 0.8 & 233.0 & 16 \\
\hline ZF-30 & 90 & 30 & 877 & 3050 & 10.5 & 76.4 & 0.4 & 76.8 & 0.6 & 90.0 & 15 \\
\hline $95-114$ & & & & & & & & & & & \\
\hline$\overline{Z A-6}$ & 24 & 52 & 548 & 2070 & 10.1 & 65.8 & 1 & 64.4 & 0.9 & 12.0 & 30 \\
\hline ZB-10 & 180 & 29 & 494 & 3080 & 11.3 & 65.7 & 0.7 & 65.7 & 1.1 & 62.0 & 25 \\
\hline ZE-20 & 164 & 16 & 222 & 4600 & 10.5 & 65.7 & 0.6 & 65.7 & 1.0 & 65.0 & 26 \\
\hline ZG-30 & 86 & 24 & 375 & 2770 & 11.5 & 65.3 & 0.5 & 65.2 & 1.0 & 61.0 & 29 \\
\hline$\underline{95-130}$ & & & & & & & & & & & \\
\hline ZA-6 & 187 & 41 & 885 & 3610 & 12 & 63.2 & 0 & 63 & 0.7 & 54 & 20 \\
\hline ZB-10 & 146 & 25 & 850 & 7190 & 12 & 63.3 & 0 & 63 & 0.6 & 60 & 16 \\
\hline ZD-20 & 190 & 70 & 1164 & 1960 & 11 & 63.3 & 0 & 63 & 0.9 & 66 & 29 \\
\hline ZF-30 & 135 & 23 & 914 & 7960 & 12 & 63.2 & 0 & 63 & 0.6 & 60 & 16 \\
\hline $98-23$ & & & & & & & & & & & \\
\hline Zaa-1 & 32 & 9 & 2791 & 5606 & 42 & 58.5 & 0 & 59 & 0.7 & 72 & 14 \\
\hline Zaa-1 & 28 & 7 & 5278 & 12,510 & 30 & 58.5 & 1 & 59 & 0.7 & 70 & 8 \\
\hline Zaa-1 & 31 & 8 & 6559 & 13,306 & 30 & 54.7 & 1 & 55 & 0.6 & 60 & 7 \\
\hline Zaa-1 & 34 & 11 & 3375 & 5685 & 27 & 55.5 & 1 & 56 & 0.8 & 64 & 15 \\
\hline Zaa-1 & 29 & 14 & 1060 & 2630 & 12 & 115.5 & 1 & 117 & 0.8 & 137 & 15 \\
\hline ZA-7 & 195 & 30 & 4868 & 15,052 & 39 & 55.4 & 2 & 56 & 1.6 & 67 & 7 \\
\hline ZC-15 & 142 & 46 & 7614 & 11,909 & 53 & 57 & 2 & 58 & 1.7 & 68 & 8 \\
\hline ZC-15 & 155 & 34 & 2318 & 5958 & 20 & 67 & 1 & 68 & 1.1 & 96 & 15 \\
\hline $\begin{array}{l}\text { ZF-30 } \\
\text { Getukti }\end{array}$ & 80 & 21 & 3291 & 6882 & 20 & 67.5 & 1 & 68 & 0.7 & 89 & 12 \\
\hline $\mathrm{ZA}-1$ & 95 & 7 & 336 & 2220 & 38 & 52 & 0 & 52 & 0.6 & 62 & 20 \\
\hline$Z A-1$ & 52 & 15 & 1180 & 2050 & 11 & 51.4 & 0 & 51 & 0.6 & 44 & 24 \\
\hline ZA-1 & 36 & 16 & 762 & 875 & 9 & 51.3 & 1 & 52 & 0.9 & 59 & 34 \\
\hline ZB-5 & 242 & 39 & 809 & 2630 & 9 & 52.4 & 0 & 53 & 0.5 & 66 & 16 \\
\hline ZB-5 & 140 & 20 & 747 & 2680 & 10 & 51.4 & 0 & 52 & 0.5 & 60 & 18 \\
\hline ZD-20 & 155 & 41 & 2425 & 5680 & 8 & 62 & 0 & 65 & 0.6 & 190 & 14 \\
\hline ZD-20 & 165 & 20 & 1396 & 7600 & 7 & 66.7 & 1 & 68 & 0.6 & 106 & 11 \\
\hline ZF-50 & 240 & 23 & 443 & 2850 & 7 & 61.9 & 0 & 64 & 0.6 & 153 & 15 \\
\hline Roussea & & & & & & & & & & & \\
\hline ZA-1 & 24 & 8 & 1195 & 2117 & 12 & 59 & 0 & 59 & 0.4 & 69 & 23 \\
\hline$Z A-1$ & 29 & 8 & 610 & 1215 & 13 & 55 & 0 & 55 & 0.6 & 73 & 34 \\
\hline ZA-1 & 24 & 10 & 942 & 1192 & 11.4 & 54.9 & 0.4 & 55 & 0.6 & 63 & 40 \\
\hline$Z A-1$ & 36 & 11 & 591 & 1042 & 13.5 & 54.2 & 0.4 & 54 & 0.6 & 63 & 44 \\
\hline ZA-1 & 34 & 4 & 523 & 2167 & 15 & 53 & 1 & 54 & 1.1 & 57 & 64 \\
\hline ZA-1 & 44 & 7 & 668 & 2254 & 14 & 53 & 1 & 53 & 0.5 & 52 & 14 \\
\hline ZA-1 & 35 & 10 & 704 & 1272 & 13 & 53 & 0 & 53 & 1.1 & 55 & 99 \\
\hline ZA-1 & 25 & 5 & 1236 & 3002 & 14 & 53 & 0 & 53 & 0.5 & 56 & 33 \\
\hline SA-8 & 482 & 2040 & 29 & 59 & 1 & 52.7 & 4 & 47 & 14 & -222 & 285 \\
\hline
\end{tabular}


TABLE 4A. PALEOGENE BATHOLITH. U-Pb (ZIRCON) GEOCHRONOLOGIC ANALYSES BY THERMAL IONIZATION MASS SPECTROMETRY (Continued)

\begin{tabular}{|c|c|c|c|c|c|c|c|c|c|c|c|}
\hline & & & & & & \multicolumn{6}{|c|}{ Apparent ages (Ma) } \\
\hline Sample & $\begin{array}{l}\text { Wt. } \\
(\mu \mathrm{g})\end{array}$ & $\begin{array}{l}\mathrm{Pb}_{\mathrm{c}} \\
(\mathrm{pg})\end{array}$ & $\begin{array}{c}\mathrm{U} \\
\text { (ppm) }\end{array}$ & $\frac{{ }^{206} \mathrm{~Pb}}{{ }^{204} \mathrm{~Pb}}$ & $\frac{{ }^{206} \mathrm{~Pb}}{{ }^{208} \mathrm{~Pb}}$ & $\frac{{ }^{206} \mathrm{~Pb}^{*}}{{ }^{238} \mathrm{U}}$ & \pm & $\frac{{ }^{207} \mathrm{~Pb}^{*}}{{ }^{235} \mathrm{U}}$ & \pm & $\frac{{ }^{207} \mathrm{~Pb}^{*}}{{ }^{206} \mathrm{~Pb}^{*}}$ & \pm \\
\hline \multicolumn{12}{|c|}{ Halibut (98-42) } \\
\hline $\mathrm{ZA}-1$ & 119 & 34 & 684 & 1243 & 12 & 51.9 & 1 & 52 & 0.8 & 61 & 38 \\
\hline$Z A-1$ & 87 & 13 & 712 & 2376 & 14 & 51.8 & 0 & 52 & 0.4 & 49 & 26 \\
\hline $\mathrm{ZA}-1$ & 53 & 9 & 1101 & 3329 & 15 & 51.4 & 0 & 52 & 0.4 & 55 & 28 \\
\hline$Z A-1$ & 64 & 12 & 942 & 2694 & 14 & 51.3 & 2 & 51 & 2.2 & 43 & 148 \\
\hline ZA-1 & 53 & 8 & 382 & 1301 & 19 & 51.3 & 1 & 51 & 0.7 & 40 & 72 \\
\hline SA-11 & 500 & 850 & 23 & 78 & 1 & 51.5 & 2 & 51 & 12 & -36 & 280 \\
\hline SA-10 & 468 & 1600 & 36 & 78 & 1 & 51.2 & 2 & 50 & 13 & -138 & 290 \\
\hline
\end{tabular}




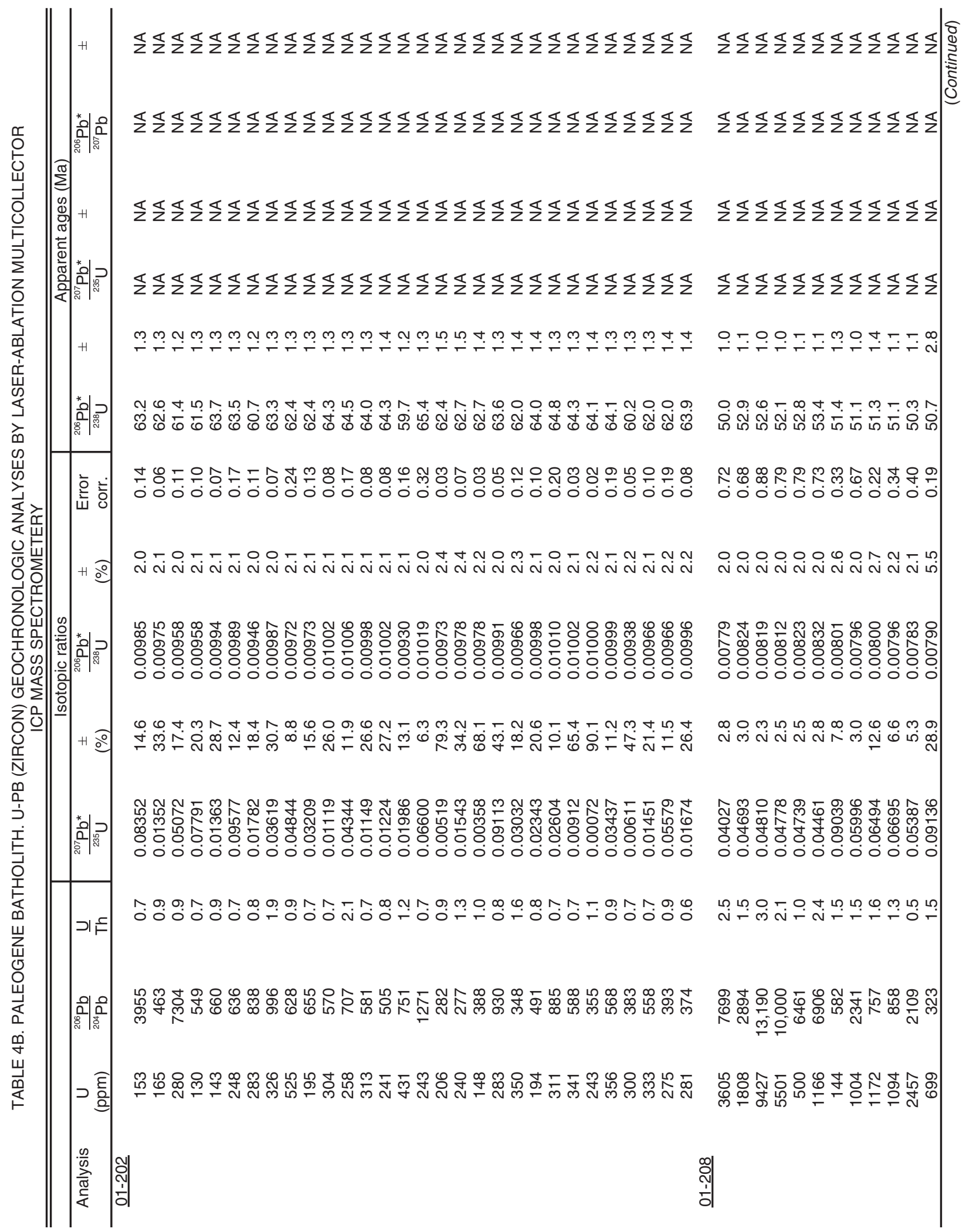




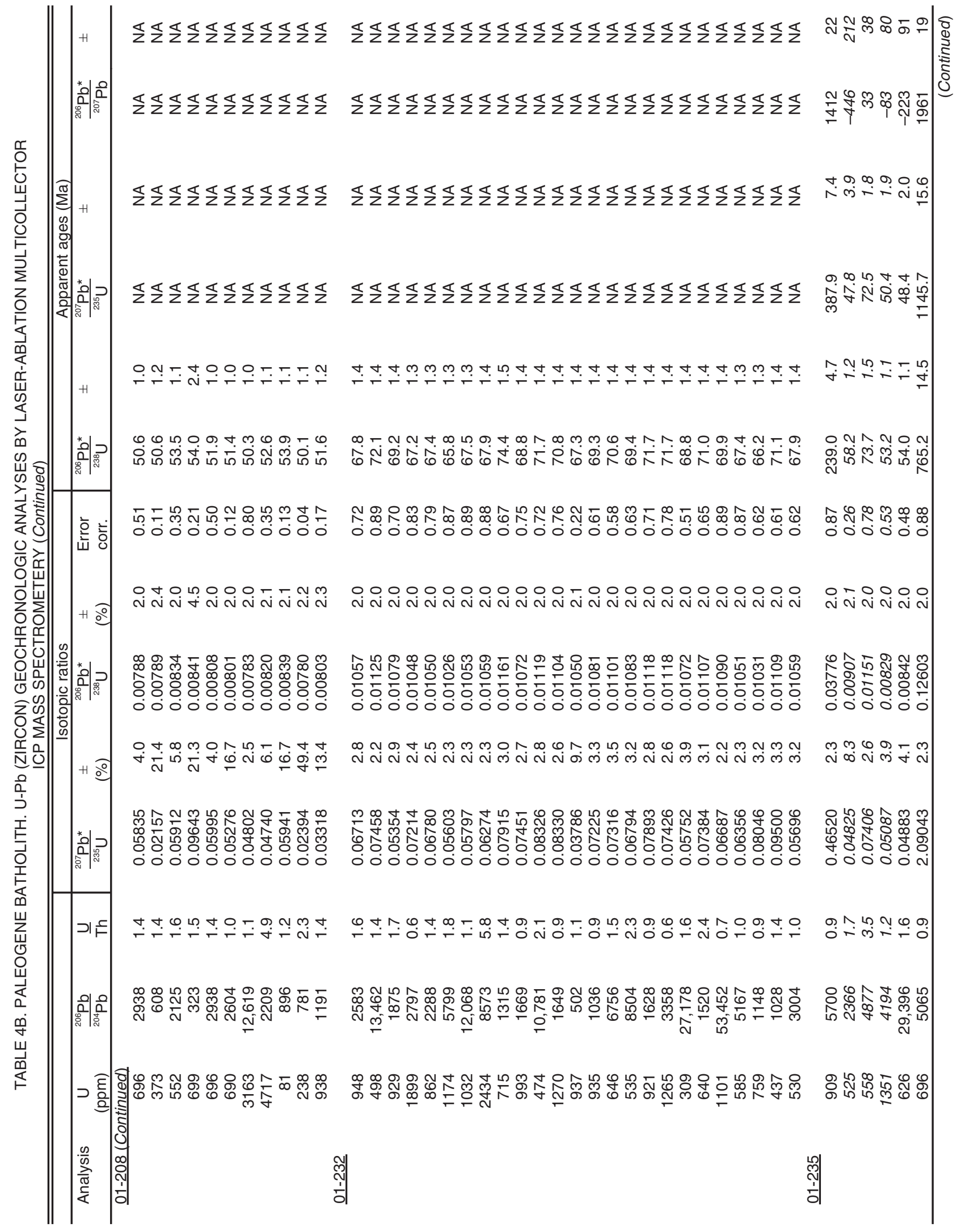




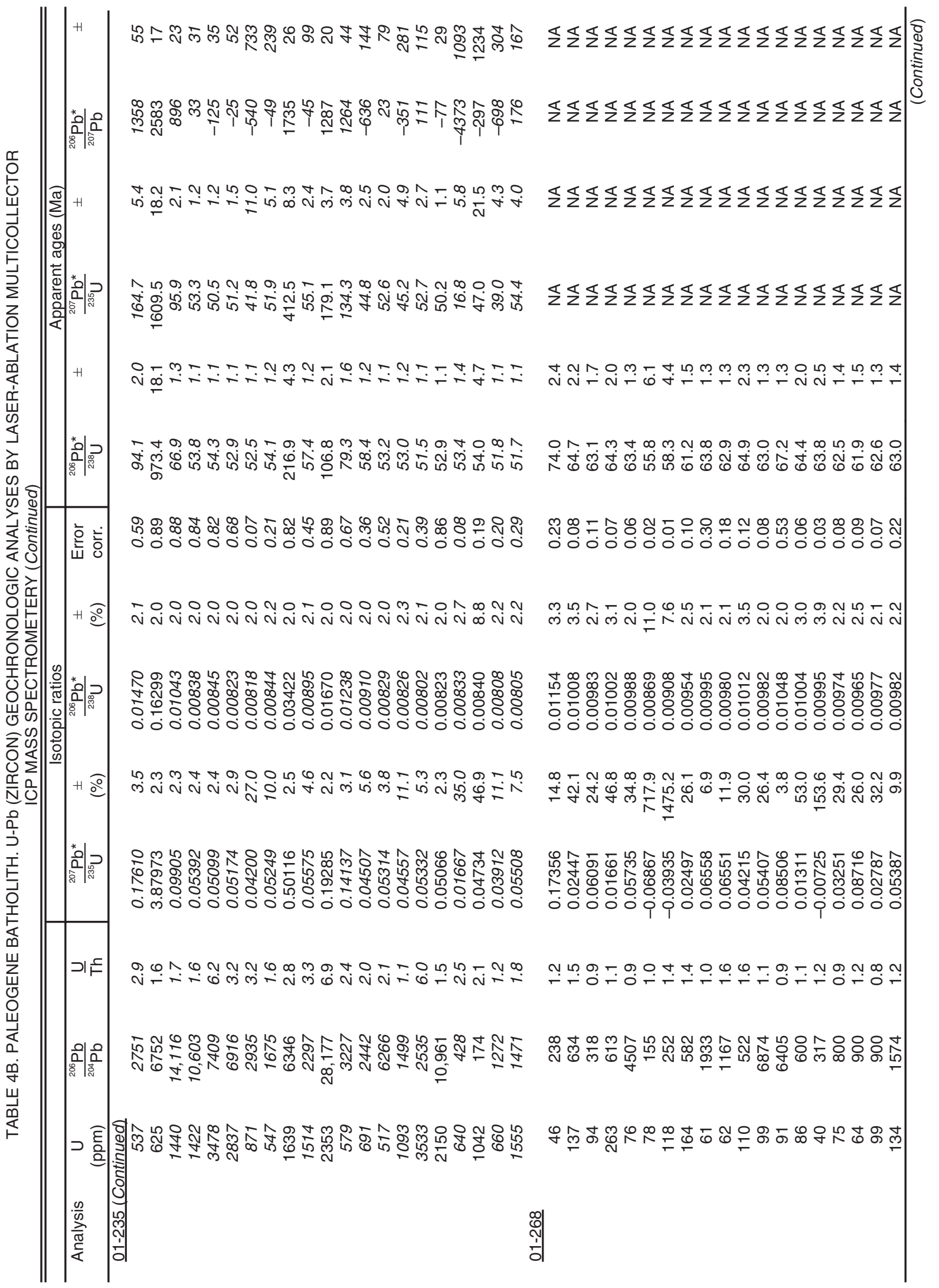




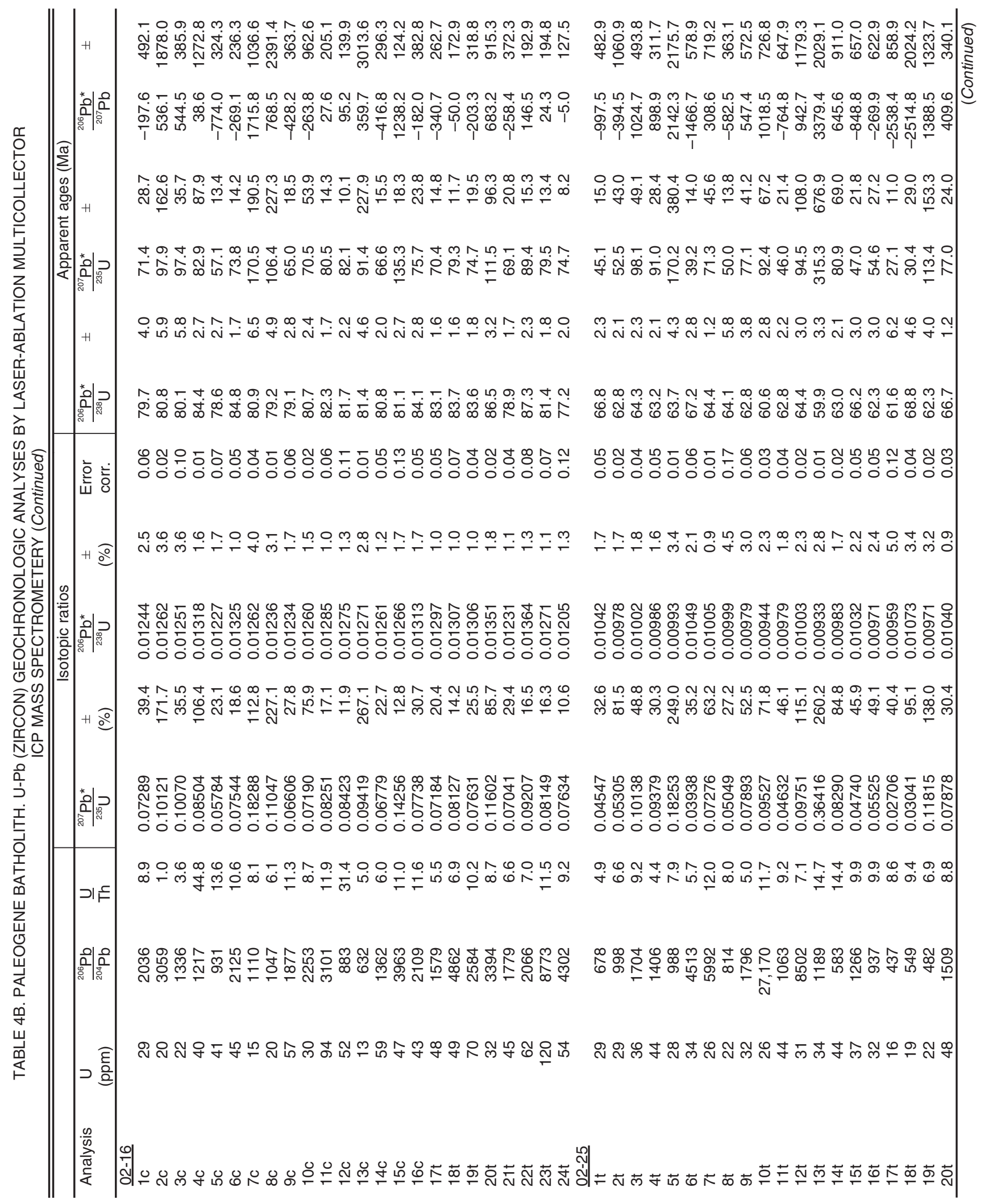




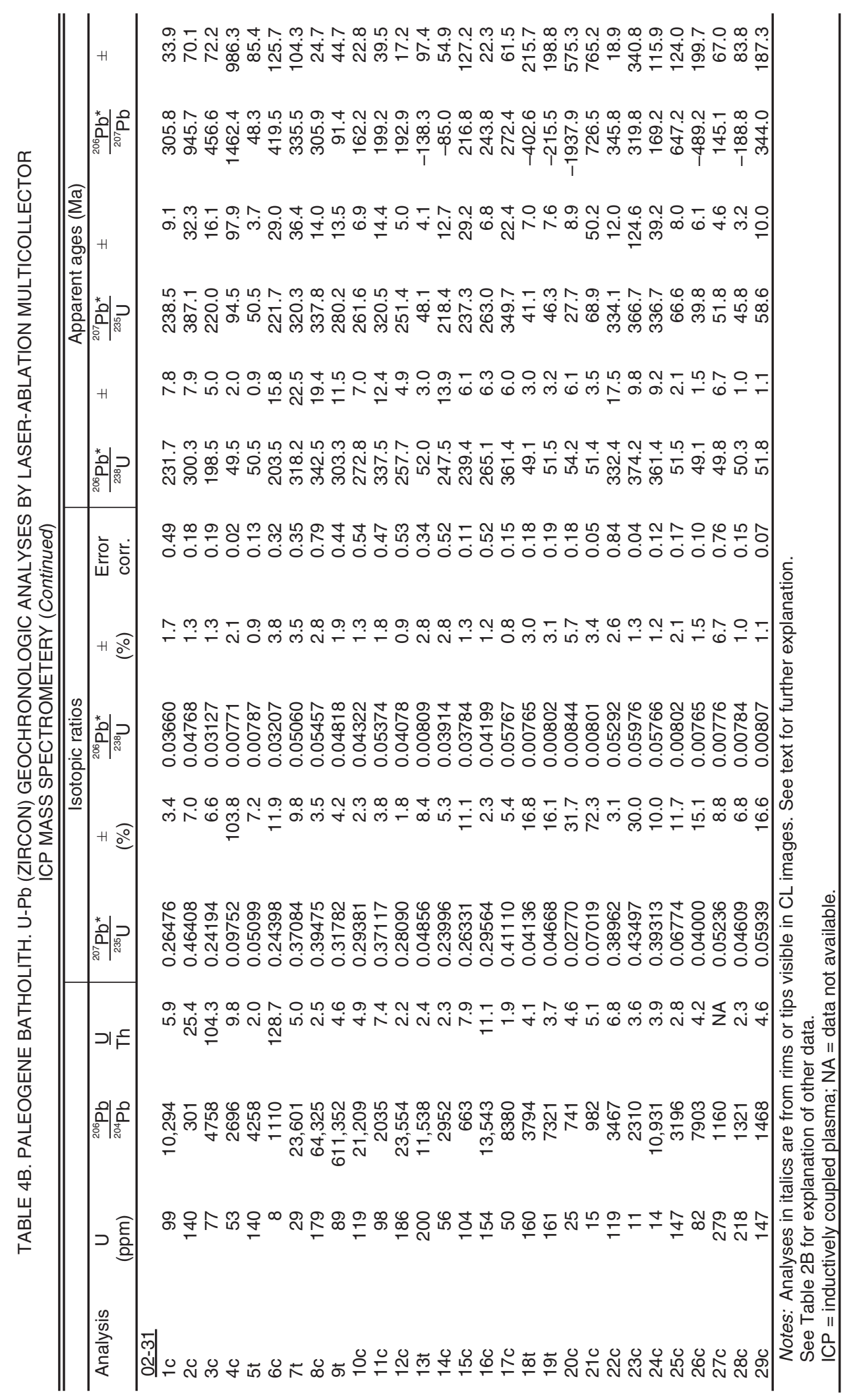




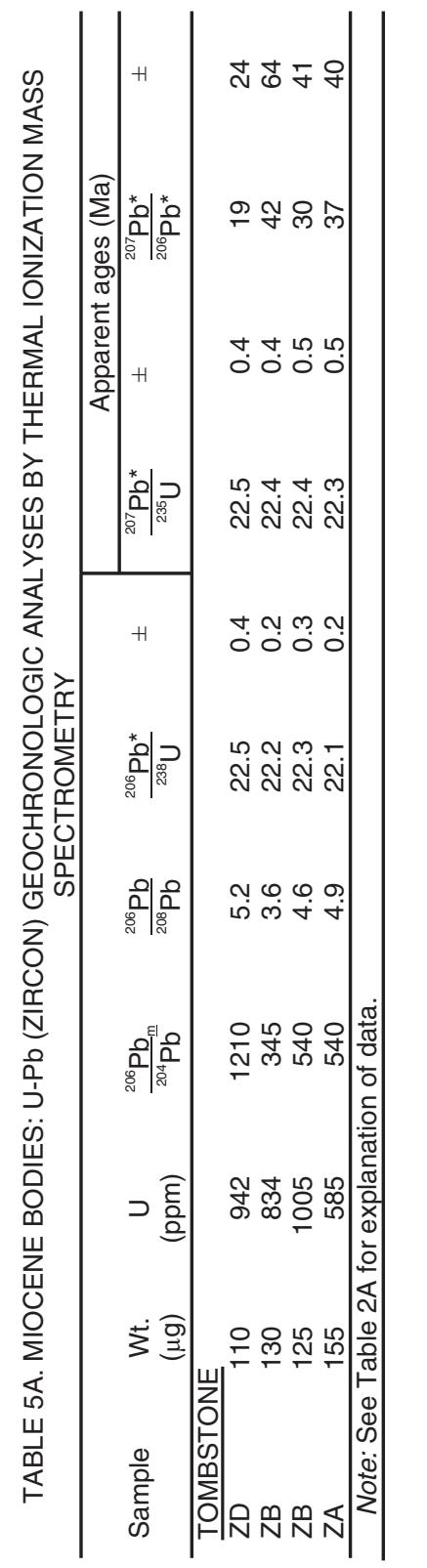

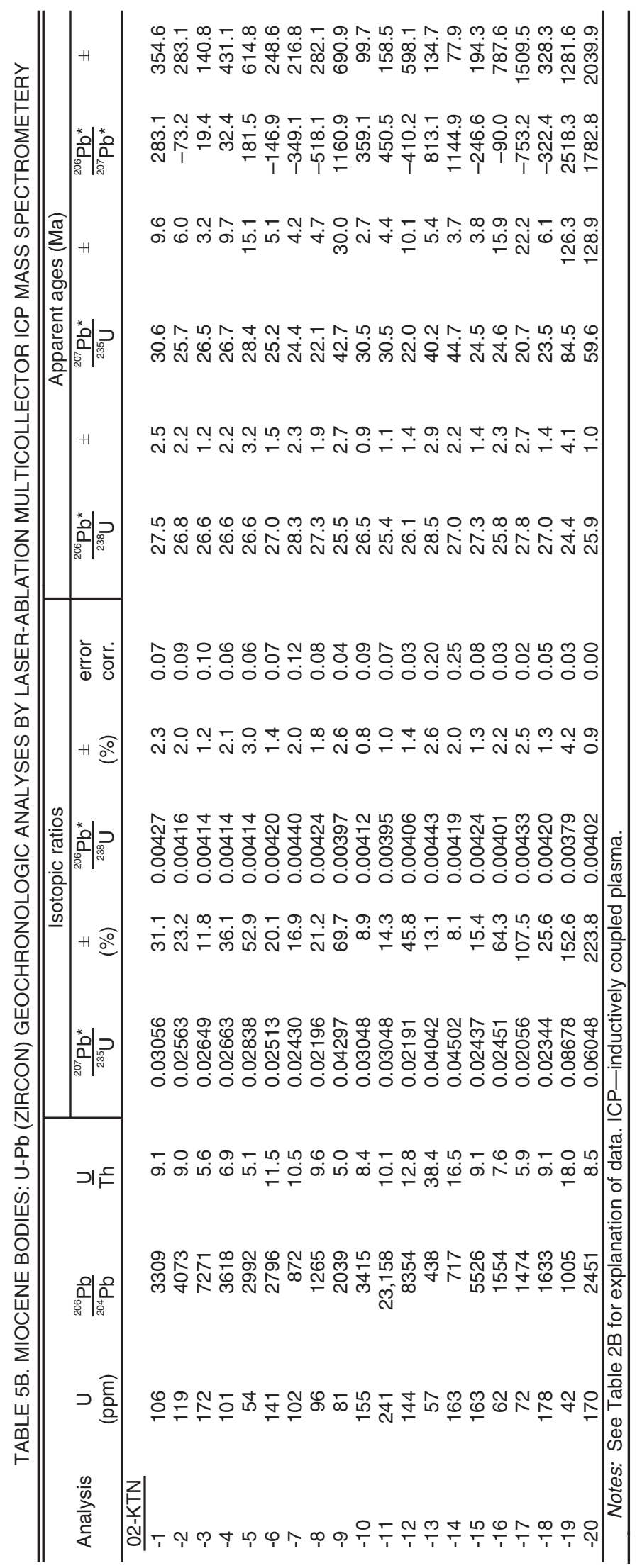


Geological Society of America Special Paper 456

Crustal Cross Sections from the Western North American Cordillera and Elsewhere: Implications for Tectonic and Petrologic Processes

Edited by Robert B. Miller and Arthur W. Snoke

(C) 2009 The Geological Society of America

To accompany Chapter 4, "Mid-Cretaceous-Recent crustal evolution in the central Coast orogen, British Columbia and southeastern Alaska," by Maria Luisa Crawford,

Keith A. Klepeis, George E. Gehrels, and Jennifer Lindline

\section{APPENDIX. METHODS}

\section{TIMS Analyses}

$\mathrm{U}-\mathrm{Pb}$ samples were processed using a jaw crusher, roller crusher, Wilfley table, heavy liquids, and Frantz magnetic separator. The non-magnetic zircons were sieved into size fractions and then selected for analysis based on optical properties using a binocular microscope. An effort was made to select grain with few fractures, inclusions, and cores, and highly elongate rods were possible. The zircons were dissolved in $\mathrm{HF}>\mathrm{HNO}_{3}$ in 0.01 $\mathrm{ml}$ Teflon microcapsules within a $125 \mathrm{ml}$ dissolution chamber during a period of $30 \mathrm{~h}$ at $245{ }^{\circ} \mathrm{C}$. The solutions were evaporated to dryness, ${ }^{205} \mathrm{~Pb} /{ }^{235-233} \mathrm{U}$ spike was added, and the precipitate was dissolved in the dissolution chamber in $3.1 \mathrm{~N} \mathrm{HCl}$ for $8 \mathrm{~h}$ at $225^{\circ} \mathrm{C}$.

Isotope analyses were conducted with a VG-354 mass spectrometer equipped with six Faraday collectors and an axial Daly detector. The measurements were made in computer-controlled dynamic mode, with the Daly detector used simultaneously with the Faraday collectors to measure ${ }^{204} \mathrm{~Pb}$. The gain factor of the Daly detector was determined continuously by comparing ${ }^{206} \mathrm{~Pb}_{\text {(Faraday) }} /{ }^{205} \mathrm{~Pb}_{\text {(Faraday) }}$ with ${ }^{206} \mathrm{~Pb}_{\text {(Faraday) }} /{ }^{205} \mathrm{~Pb}_{\text {(Daly) }}$ and ${ }^{206} \mathrm{~Pb}_{\text {(Faraday) }} /$ ${ }^{207} \mathrm{~Pb}_{\text {(Faraday) }}$ with ${ }^{206} \mathrm{~Pb}_{\text {(Faraday) }} /{ }^{207} \mathrm{~Pb}$ (Daly). Analytical methods are reported by Gehrels et al. (1991), and isotopic data were processed utilizing data reduction and plotting programs of Ludwig (1991a, 1991b).

All uncertainties are at the $95 \%$ confidence level.

$*$ radiogenic $\mathrm{Pb}$

Z-zircon, S-Sphene

$\mathrm{Pb}_{\mathrm{c}}$-total common $\mathrm{Pb}$ in picograms

${ }^{206} \mathrm{~Pb}_{\mathrm{m}}{ }^{204} \mathrm{~Pb}$ is measured ratio, uncorrected for spike, fractionation, or common $\mathrm{Pb}$.

${ }^{206} \mathrm{~Pb} /{ }^{208} \mathrm{~Pb}$ is corrected for blank, spike, fractionation, and initial $\mathrm{Pb}$.

$\mathrm{Pb}$ and $\mathrm{U}$ concentrations have uncertainties of up to $25 \%$ due to uncertainty in grain weight.

Constants used: $\lambda_{235}=9.8485 \times 10^{-10}, \lambda_{238}=1.55125 \times 10^{-10}$, ${ }^{238} \mathrm{U} /{ }^{235} \mathrm{U}=137.88$.

In calculating $U$ concentration and apparent ages, the isotope ratios are adjusted as follows:

(1) Mass dependent corrections factors of: $0.14 \pm 0.06 \% /$ $\mathrm{AMU}$ for $\mathrm{Pb}$ and $0.04 \pm 0.04 \% / \mathrm{AMU}$ for $\mathrm{UO}_{2}$.
(2) $\mathrm{Pb}$ ratios corrected for $0.010 \pm 0.005$ ng blank with ${ }^{206} \mathrm{~Pb} /{ }^{204} \mathrm{~Pb}=18.6 \pm 0.3,{ }^{207} \mathrm{~Pb} /{ }^{204} \mathrm{~Pb}=15.5 \pm 0.3$, and ${ }^{208} \mathrm{~Pb} / 204 \mathrm{~Pb}=38.0 \pm 0.8$.

(3) U has been adjusted for $0.001 \pm 0.001 \mathrm{ng}$ blank.

(4) Initial $\mathrm{Pb}$ composition is from Stacey and Kramers (1975), with uncertainties of 2.0 for ${ }^{206} \mathrm{~Pb} /{ }^{204} \mathrm{~Pb}, 0.3$ for ${ }^{207} \mathrm{~Pb} /{ }^{204} \mathrm{~Pb}$, and 2.0 for ${ }^{208} \mathrm{~Pb} /{ }^{204} \mathrm{~Pb}$.

All analyses conducted using conventional isotope dilution and thermal ionization mass spectrometry, as described by Gehrels et al. (1991).

Isotopic data were processed with programs of Ludwig (1991a, 1991b).

\section{ICP Analyses}

\section{${ }^{206} \mathrm{~Pb} /{ }^{204} \mathrm{~Pb}$ is measured ratio.}

All uncertainties are at the 1-sigma level, and include only measurement errors.

$\mathrm{U}$ concentration and $\mathrm{U} / \mathrm{Th}$ have uncertainties of $\sim 25 \%$.

Decay constants: ${ }^{235} \mathrm{U}=9.8485 \times 10^{-10},{ }^{238} \mathrm{U}=1.55125 \times 10^{-10}$, ${ }^{238} \mathrm{U} /{ }^{235} \mathrm{U}=137.88$.

Isotope ratios are corrected for $\mathrm{Pb} / \mathrm{U}$ fractionation by comparison with standard zircon with an age of $564 \pm 4 \mathrm{Ma}$.

Initial $\mathrm{Pb}$ composition interpreted from Stacey and Kramers (1975), with uncertainties of 1.0 for ${ }^{206} \mathrm{~Pb} /{ }^{204} \mathrm{~Pb}$ and 0.3 for ${ }^{207} \mathrm{~Pb} /{ }^{204} \mathrm{~Pb}$.

\section{REFERENCES CITED}

Aleinikoff, J.N., Schenck, W.S., Plank, M.O., Srogi, L., Fanning, C.M., Kamo, S.L., and Bosbyshell, H., 2006, Deciphering igneous and metamorphic events in high-grade rocks of the Wilmington Complex, Delaware: Morphology, cathodoluminescence and backscattered electron zoning, and SHRIMP U-Pb geochronology of zircon and monazite: Geological Society of America Bulletin, v. 118, p. 39-64.

Gehrels, G.E., 2000, Introduction to detrital zircon studies of Paleozoic and Triassic strata in western Nevada and northern California, in Soreghan, M.J., and Gehrels, G.E., eds., Paleozoic and Triassic paleogeography and tectonics of western Nevada and northern California: Geological Society of America Special Paper 347, p. 1-18.

Gehrels, G.E., McClelland, W.C., Samson, S.D., and Patchett, P.J., 1991, U-Pb geochronology of detrital zircons from a continental margin assemblage in the northern Coast Mountains, southeastern Alaska: Canadian Journal of Earth Sciences, v. 28, p. 1285-1300.

Ludwig, K.R., 1991a, A computer program for processing $\mathrm{Pb}-\mathrm{U}-\mathrm{Th}$ isotopic data: U.S. Geological Survey Open-File Report 88-542, 37 p.

Ludwig, K.R., 1991b, A plotting and regression program for radiogenicisotopic data: U.S. Geological Survey Open-File Report 91-445, 39 p.

Stacey, J.S., and Kramers, J.D., 1975, Approximation of terrestrial lead isotope evolution by a two-stage model: Earth and Planetary Science Letters, v. 26, p. 207-221, doi: 10.1016/0012-821X(75)90088-6. 